\title{
Performance of a first generation X-band photoelectron rf gun
}

\author{
C. Limborg-Deprey, ${ }^{*}$ C. Adolphsen, D. McCormick, M. Dunning, K. Jobe, H. Li, \\ T. Raubenheimer, A. Vrielink, T. Vecchione, F. Wang, and S. Weathersby \\ SLAC National Accelerator Laboratory, Menlo Park, California 94025, USA
}

(Received 24 August 2015; published 4 May 2016)

\begin{abstract}
Building more compact accelerators to deliver high brightness electron beams for the generation of high flux, highly coherent radiation is a priority for the photon science community. A relatively straightforward reduction in footprint can be achieved by using high-gradient X-band $(11.4 \mathrm{GHz})$ rf technology. To this end, an X-band injector consisting of a 5.5 cell $\mathrm{rf}$ gun and a 1-m long linac has been commissioned at SLAC. It delivers an $85 \mathrm{MeV}$ electron beam with peak brightness somewhat better than that achieved in S-band photoinjectors, such as the one developed for the Linac Coherent Light Source (LCLS). The X-band rf gun operates with up to a $200 \mathrm{MV} / \mathrm{m}$ peak field on the cathode, and has been used to produce bunches of a few $\mathrm{pC}$ to $1.2 \mathrm{nC}$ in charge. Notably, bunch lengths as short as $120 \mathrm{fs}$ rms have been measured for charges of $5 \mathrm{pC}\left(\sim 3 \times 10^{7}\right.$ electrons), and normalized transverse emittances as small as $0.22 \mathrm{~mm}-\mathrm{mrad}$ have been measured for this same charge level. Bunch lengths as short as 400 (250) fs rms have been achieved for electron bunches of $100(20) \mathrm{pC}$ with transverse normalized emittances of $0.7(0.35) \mathrm{mm}$-mrad. We report on the performance and the lessons learned from the operation and optimization of this first generation X-band gun.
\end{abstract}

DOI: 10.1103/PhysRevAccelBeams.19.053401

\section{INTRODUCTION}

There has long been an interest in having compact electron sources for a variety of accelerator applications such as FELs, electron scattering experiments, imaging and radiation therapy. In particular, large-scale X-ray Free Electron Lasers (XFELs), which are the ultimate tunable microscopes to probe matter on the atomic scale, have drive linacs that range from a few hundreds of meters to about kilometer in length. Currently, they are based on three rf technologies: $1.3 \mathrm{GHz}, 24 \mathrm{MV} / \mathrm{m}$, superconducting L-Band [1], $2.86 \mathrm{GHz}, 17 \mathrm{MV} / \mathrm{m}$, room-temperature S-Band [2-4], and $5.7 \mathrm{GHz}, 35 \mathrm{MV} / \mathrm{m}$, room temperature C-Band at SACLA [5] and SwissFEL [6].

For the future, there is interest in reducing the linac length to the 100-m level [7-9] by using room temperature, high gradient (70-100 MV/m), $11.4 \mathrm{GHz} \mathrm{X}-$ Band technology that was developed for the NLC/GLC programs in the 1990's and early 2000's [10] while still delivering the high brightness electrons needed to produce high peak intensity photon bunches [11]. Other promising very-high gradient $(1-10 \mathrm{GV} / \mathrm{m})$ accelerator technologies-laser generated plasma wakefield acceleration [12] and beam generated plasma acceleration [13] — are being developed. The beam quality, reproducibility and stability are however still far from that needed to drive FELs.

\footnotetext{
*limborg@slac.stanford.edu

Published by the American Physical Society under the terms of the Creative Commons Attribution 3.0 License. Further distribution of this work must maintain attribution to the author(s) and the published article's title, journal citation, and DOI.
}

As an offshoot of the $\sim 100 \mathrm{M} \$ \mathrm{X}$-band program at SLAC, an X-band gun was built in the mid-2000s for a compact Compton source that was to be used for a medical application [14]. This was done during a period when the LCLS S-band gun was being developed and there was much interest in photoinjectors based on pulsed rf technology. This first $\mathrm{X}$-band gun operated with up to a $200 \mathrm{MV} / \mathrm{m}$ cathode peak field, but a detailed beam characterization was never performed as the program ended early.

In the late 2000s, there was renewed interest in X-band technology from the experience with the $\mathrm{X}$-band harmonic linearizer used at LCLS and planned elsewhere $[15,16]$, the desire by laboratories to fit next generation light sources into existing lab space $[17,18]$ and applications for compact gamma-ray Compton sources [19]. However, unlike S-band and C-band technology at that time, commercial high power klystrons were not available (one company makes them today), and a low-emittance X-band demonstration linac did not exist. To address this second issue, that is, showing that high gradient acceleration and low emittance bunches are simultaneously achievable, an all X-band injector was built in the Next Linear Collider Test Area (NLCTA) at SLAC, which has four high power X-band sources (each producing up to $200 \mathrm{MW}, 250 \mathrm{~ns}$ long pulses). Called the X-Band Test Area (XTA), this accelerator runs parallel to the original beamline at the downstream end of the accelerator enclosure, but it is only about $7 \mathrm{~m}$ long.

The rf gun presently in operation at XTA is denoted "Mark- 0, , and is basically a copy of the 5.5 cell original 
design (using leftover parts), but with a race-track shaped coupling cell to minimize undesired quadrupole head-tail effects in the electron bunch. The idea was to use this gun to debug the XTA before a new, more optimized design, call the Mark-1 (see Fig. 1) was deployed. The new gun design was developed in collaboration with LLNL, and has 5.6 cells, a race-rack coupler, elliptical contoured irises, an rf coupling that better balances pulsed heating with cavity fill time and a larger mode separation [20]. Two Mark-1 guns were built, one that was to be used in XTA (but now would require additional funding to test), and one that was operated at LLNL, although without optimizing the beam emittance [19]. In this paper, we report on the performance of the Mark- 0 gun at XTA.

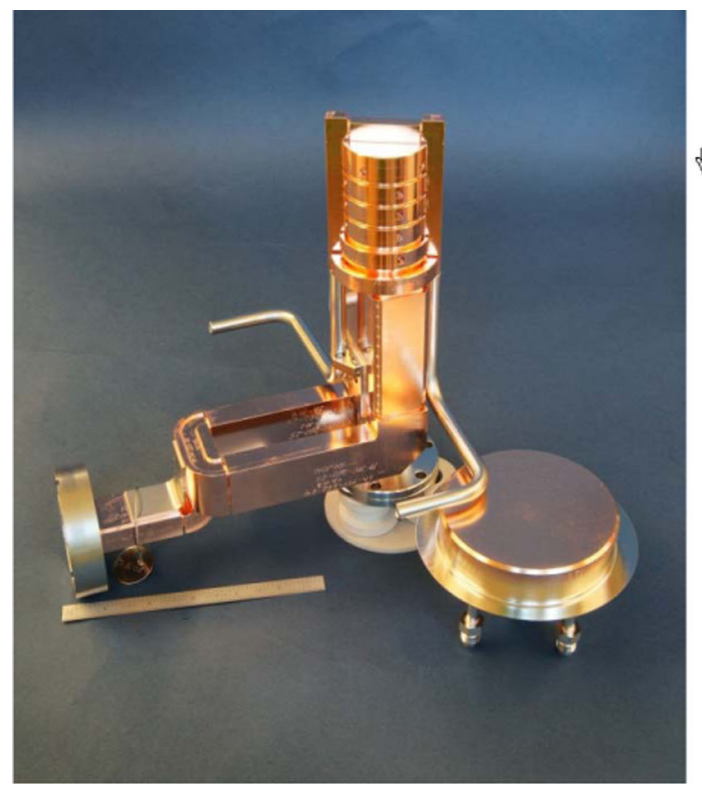

(a)

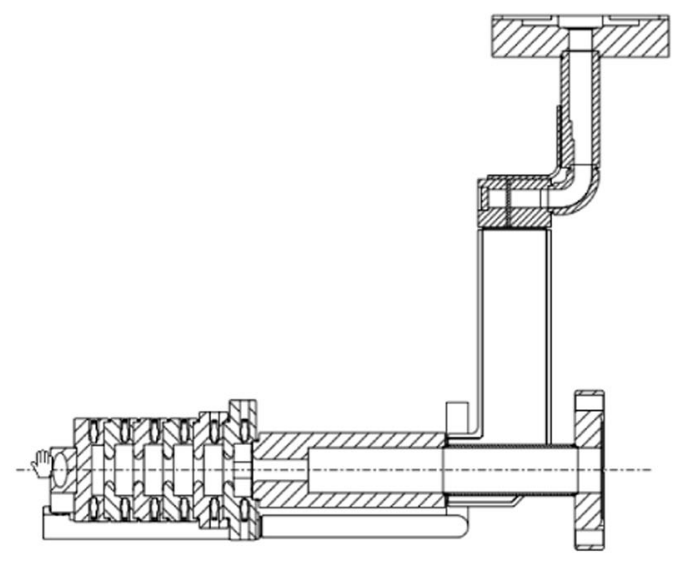

(c)
Besides providing a general demonstration of low emittance, high gradient acceleration, the XTA was also to serve as a test area for X-band rf guns targeted for compact FELs and Inverse Compton Scattering sources. Calculations indicate that the maximum peak brightness from an X-band gun with a peak cathode field of $200 \mathrm{MV} / \mathrm{m}$ is close to an order of magnitude larger than that from the standard $2.9 \mathrm{GHz}$ S-band technology where the gun typically operates with a peak acceleration field of $120 \mathrm{MV} / \mathrm{m}$. This improvement comes from a reduction of the bunch length by a factor of three to four and from a reduction in the product of the horizontal and vertical emittances by another factor of about two. Note that the cathode field during photo-emission in the LCLS gun,

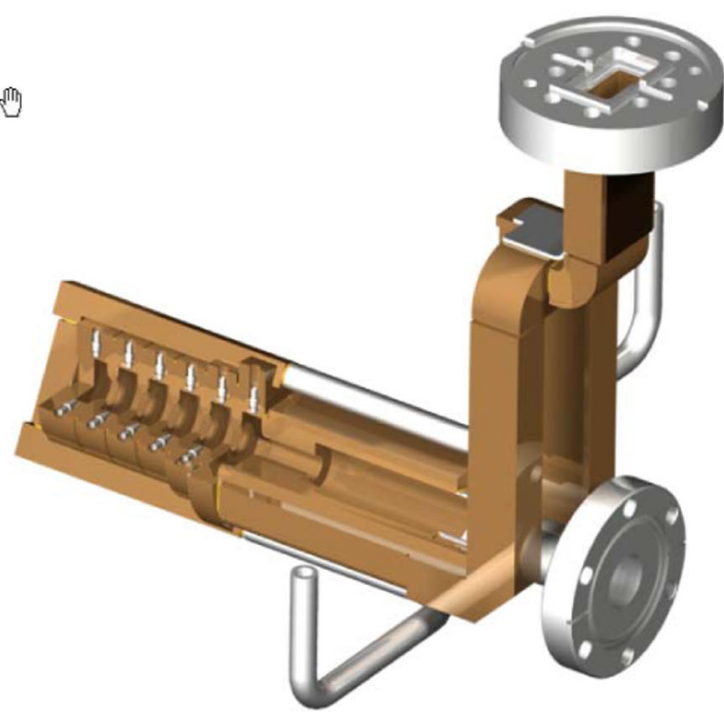

(b)

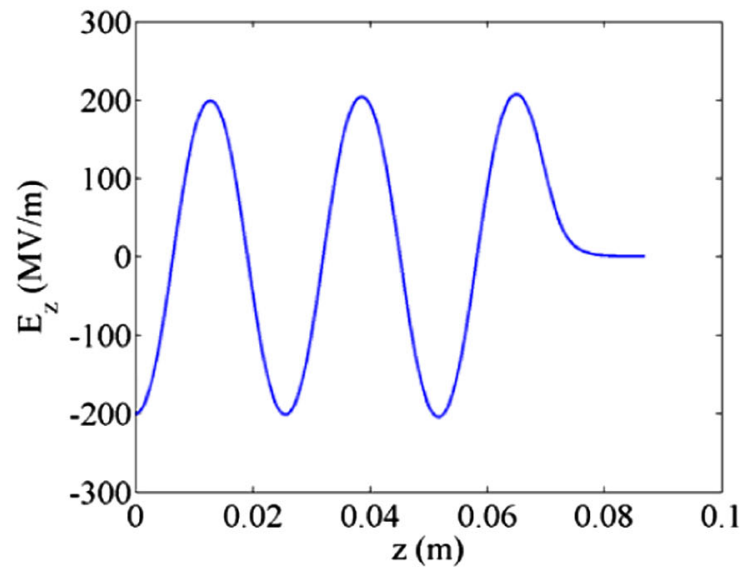

(d)

FIG. 1. Mark-1 5.6 cell X-band gun. (a) Photo of the X-band gun: an S-band gun cathode is shown on the right for comparison, (b) CAD model of the gun, (c) cross section of the gun (d) the pi-mode longitudinal field $\left(\mathrm{E}_{\mathrm{z}}\right)$ along the axis. 


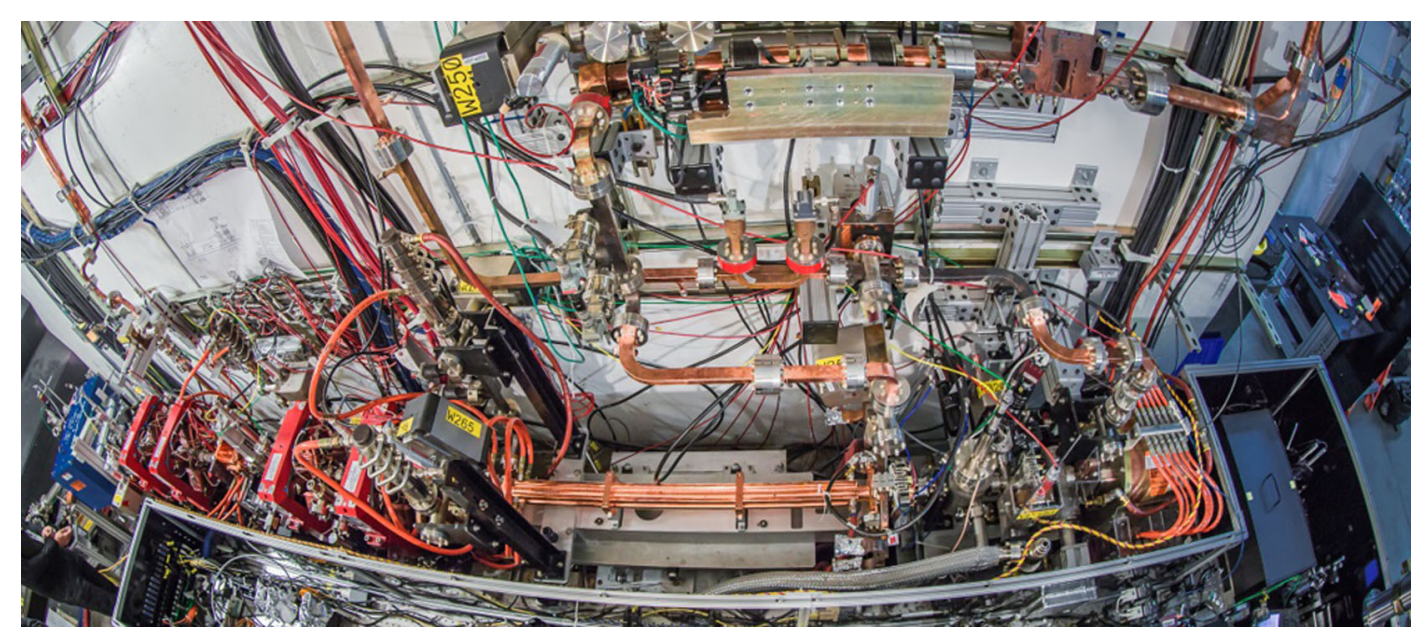

FIG. 2. X-Band test area beamline. The $1.05 \mathrm{~m}$ long copper accelerator structure is seen in the middle of the photo, and one of three phase shifters is visible on the wall. The apparent curvature of the beamline is an artefact of the wide-view camera lens.

which represents the state-of-the-art in S-band guns, is $60 \mathrm{MV} / \mathrm{m}$ at an injection phase of 30 degrees from the zero-crossing. For the "Mark-0" X-band gun, the launch phase is 50 degrees and the field at emission is $150 \mathrm{MV} / \mathrm{m}$.

\section{THE XTA}

\section{A. The rf gun}

The basic geometry of the Mark-0 gun is the same as that for the Mark-1 gun. A Computer Aided Design (CAD) model is shown in Fig. 1(b). Two input power coupling ports are located 180 degrees apart on the sides of the downstream most cell. The shape of the inner contour of this cell has been modified from cylindrical to that of a racetrack to restore at best the cylindrical symmetry of the electromagnetic fields. This compensation in the geometry suppresses the undesired quadrupole mode which would otherwise degrade the emittance. A profile of the longitudinal electric field $\left(\mathrm{E}_{z}\right)$ along the symmetry axis is plotted in Fig. 1(c) for the pi-mode, which is used for capture and acceleration. The next nearest mode frequency is $12 \mathrm{MHz}$ and $25 \mathrm{MHz}$ away from the pi-mode frequency, respectively, for the Mark-0 and the Mark-1 guns.

The cathode is brazed to the first cell, which is $0.5 / 0.6$ as long as the standard cells in the Mark-0/Mark-1 guns. The cathode is consequently non-demountable. The experimental data reported in this paper were taken with the Mark-0 gun. A water cooling channel is brazed to the back of the cathode and is visible in Fig. 1(a). The copper cathode diameter is consequently as large as the cell internal diameter of $21.8 \mathrm{~mm}$. However the irises separating the cells limit the aperture along the gun to $8 \mathrm{~mm}$, and the cathode region accessible by the laser is limited to a disk of approximately $7 \mathrm{~mm}$.

\section{B. The XTA beamline and power distribution}

A photo of the XTA is shown in Fig. 2 and a schematic is given in Fig. 3. To power the XTA, a single rf source, an
XL4 klystron, is utilized to deliver power both to the rf gun, where the electrons are generated and first accelerated, and to the single accelerator structure. The klystron provides up to $50 \mathrm{MW}$ of X-band rf power in a $1.5 \mu$ s long pulse, which is compressed in $40 \mathrm{~m}$ long SLED-II lines [21] up to $200 \mathrm{MW}$ in a $250 \mathrm{~ns}$ long pulse. The maximum klystron repetition rate is $60 \mathrm{~Hz}$, although we generally ran at $10 \mathrm{~Hz}$ to limit beam-induced radiation.

Approximately $20 \%$ of the power is attenuated in a circular-waveguide transport line that runs from the SLED-II output on the roof of the NLCTA enclosure to the XTA beamline. This power is then split via a $6 \mathrm{~dB}$ hybrid with $75 \%$ going to the accelerator structure and 25\% to an adjustable power splitter before the gun. A high power, remotely-controllable, TE01-mode phase shifter with a 90 degree phase adjustment range is located in

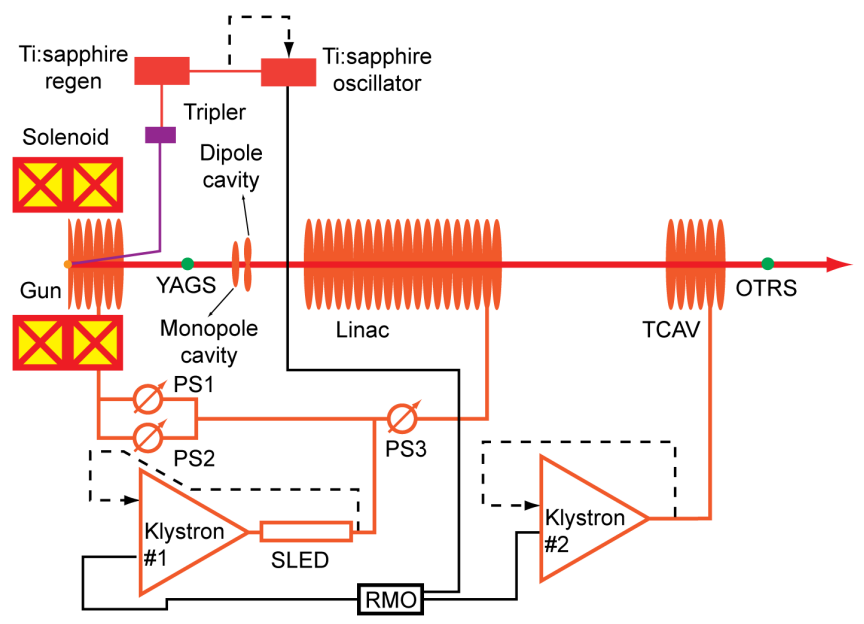

FIG. 3. Schematic of the XTA. PS1, 2, and 3 are rf phase shifters, YAGS is an yttrium aluminum garnet screen, OTRS is an optical transition radiation screen, TCAV is an X-band deflecting cavity (powered by a different RF source) and RMO is the RF master oscillator. 


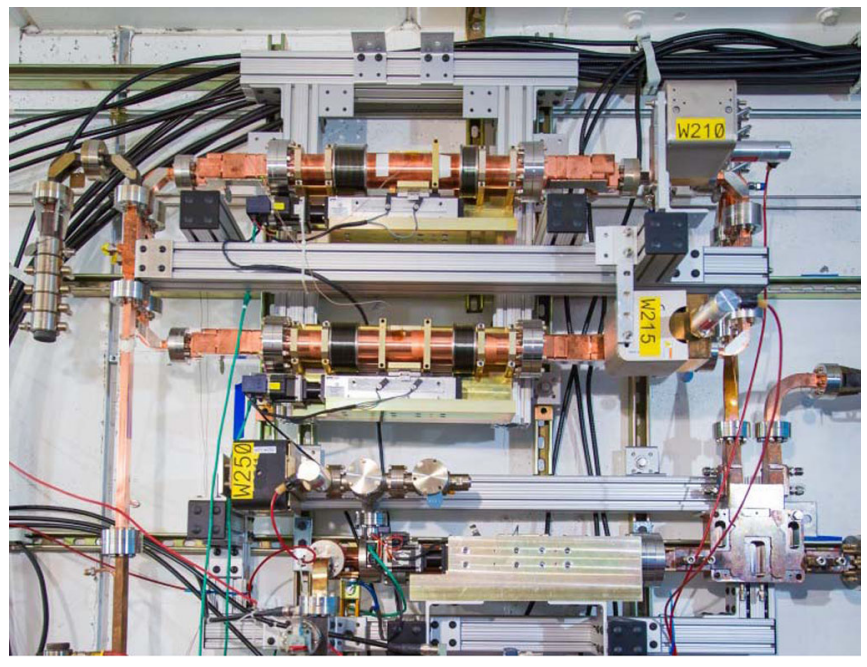

FIG. 4. Three high power phase shifters: the top two (copper cylinders with bellows on each end) are used to adjust the power going to the gun, and the third phase shifter (below the other two) is installed on the branch line that feeds the linac structure (in the photo, it is obscured by a metal support plate).

the transmission line that powers the accelerator. In the transmission line feeding the gun, the power is first split by means of a $3 \mathrm{~dB}$ hybrid and then each half passes through a phase shifter of the same design as used for the accelerator. These two lines recombine by means of another $3 \mathrm{~dB}$ hybrid and the power is transported to the gun. These two phase shifters can also be remotely controlled, allowing the phase and amplitude of the gun rf to be varied over a range of 90 degrees in phase and more than $20 \mathrm{~dB}$ in power (the unused power is absorbed in a load). The three phase shifters are shown in Fig. 4.
The transmission loss from the splitter to the gun is expected to be about $15 \%$, so a maximum of $17 \%$ of the SLED-II output power can reach the gun. To produce a peak field of $200 \mathrm{M} / \mathrm{m}$ on the cathode with a $180 \mathrm{~ns}$ square input pulse, $17 \mathrm{MW}$ is required at the input to the gun. We tune the gun line phase shifters to achieve the desired cathode field with typically $140 \mathrm{MW}$ to $180 \mathrm{MW}$ of SLED-II output power, of which $75 \mathrm{MW}$ to $97 \mathrm{MW}$ powers the $1.05 \mathrm{~m}$ long, 'T105' accelerator structure for the subsequent bunch acceleration. Generation and acceleration of photoelectron bunches up to $85 \mathrm{MeV}$ have been demonstrated in the gun plus accelerator section, which is only $1.8 \mathrm{~m}$ long.

Inferring the cathode gradient based on the SLED-II power has much uncertainty due to unknown $\mathrm{rf}$ mismatches in the waveguides runs, setting accuracy of the gun phase shifters and nonideal $\mathrm{rf}$ pulse shapes. As discussed in Sec. IV B 2, comparing laser-to-beam relative-time-ofarrival (RTOA) measurements to predications is probably the best calibration of the cathode field in the gun (given the absence of a dedicated high resolution spectrometer at the gun exit) and indicates that a 15.3 MW power at the gun input, available from a 150 MW SLED-II pulse in our system, produces a $190 \mathrm{MV} / \mathrm{m}$ cathode peak field.

\section{Laser and cathode quantum efficiency}

The XTA laser system consists of an Elite regenerative amplifier from Coherent that is fed by a Ti:Sa Tsunami oscillator from Spectra Physics. It delivers $4 \mathrm{~mJ}$ IR pulses at $600 \mathrm{~Hz}$. After recompression and conversion to UV at $266 \mathrm{~nm}$, pulses of up to $300 \mu \mathrm{J}$ were available on the laser table adjacent to the accelerator. A high reflectivity mirror was used to direct the 50 to $60 \mathrm{fs}$ long pulses to the cathode

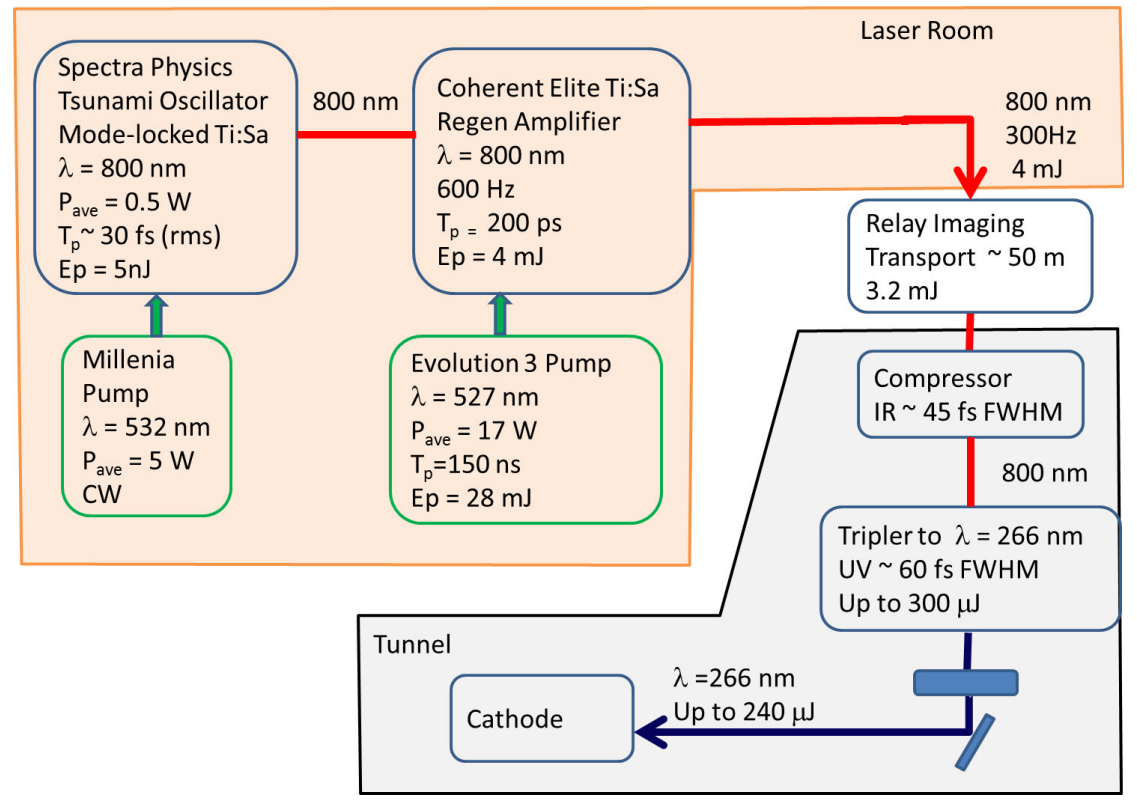

FIG. 5. Schematic of XTA laser system. 
where the pulse energy was as high as $240 \mu \mathrm{J}$. A schematic of the laser system is shown in Fig. 5.

The highly energetic laser pulses had to be used with caution - they damaged optics and accelerator components on several occasions. Having higher quantum efficiency (QE) would have facilitated the operation. With a nondemountable cathode, laser cleaning of the cathode surface would have been the most appropriate means to improve the QE. However, given the risk involved, this was not attempted.

The QE of the Mark-0 gun cathode was relatively low with values of $2 \times 10^{-5}$ at best in the good regions of the cathode. However, it was large enough to commission the rf gun and beamline and generate bunches ranging from a few $\mathrm{pC}$ up to $1.2 \mathrm{nC}$.

\section{BEAM DYNAMICS IN THE X-BAND GUN}

The advantages of using high gradient fields in the gun and during the initial acceleration of the electrons are that the charge density on the cathode surface can be higher and the electrons will reach relativistic velocities more rapidly, reducing the effects of space charge forces. Simulations show that these benefits can increase the peak bunch brightness by close to an order of magnitude when using $\mathrm{X}$-band technology compared with S-band technology.

\section{A. Advantages of a high cathode field gradient}

In Fig. 6(a), the increase in the energy of an electron in a gun (expressed by $\gamma$ ) is shown during the first 33 ps of acceleration, during which the electrons travel several $\mathrm{mm}$ beyond the cathode. Also shown in Fig. 6(b) are the fields witnessed by the electron and its longitudinal position versus time. For this calculation, the S-band gun is assumed to operate with a $120 \mathrm{MV} / \mathrm{m}$ cathode field and the X-band gun with a $200 \mathrm{MV} / \mathrm{m}$ field, which are near the upper limits for reliable operation at these two frequencies (at these field levels, breakdown events occur rarely). These field values are assumed in the following discussion unless noted otherwise. As described in [22], the transverse space charge force has a $1 / \gamma^{2}$ dependence and the primary detrimental space charge effect occurs in the region where $\gamma$ is less than about two. As seen in Fig. 6(a), $\gamma$ reaches this level roughly twice as fast in the X-band case.

\section{B. Beam dynamics in the presence of space charge}

Due to the rapid increase in $\gamma$ the bunch length is frozen 1-3 $\mathrm{mm}$ downstream of the cathode, approximately $1 \mathrm{~mm}$ for low space charge and $3 \mathrm{~mm}$ for high space charge. For negligible or low space charge, the electron bunch length depends strongly on the initial laser-to-rf phase and the final bunch lengths are similar for X-band and S-band.
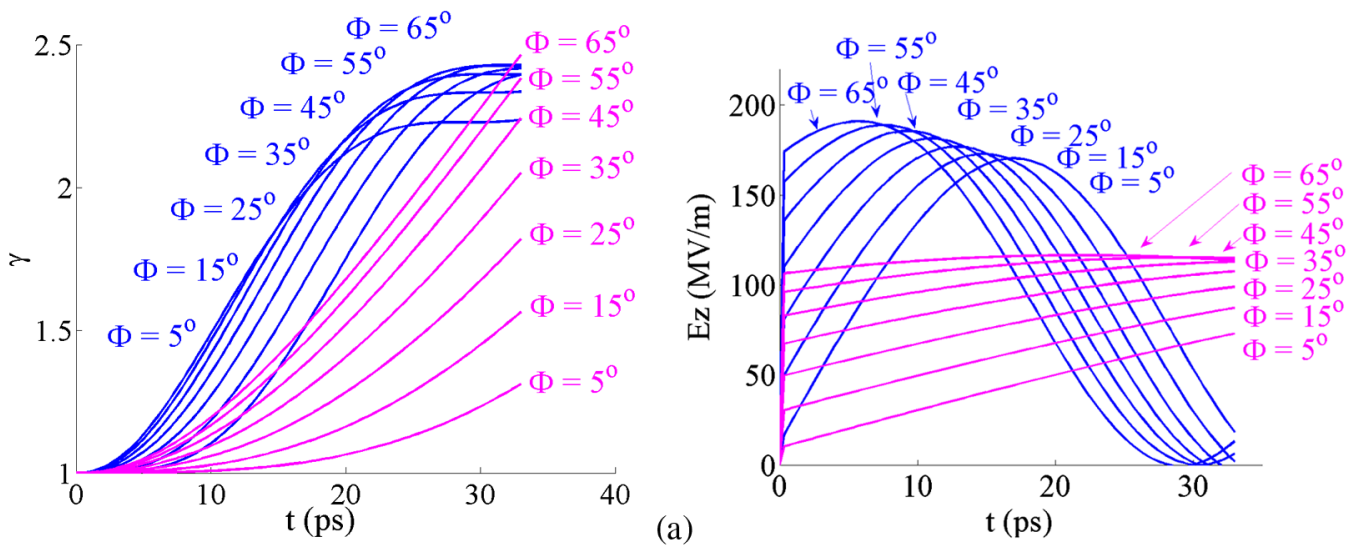

(b)

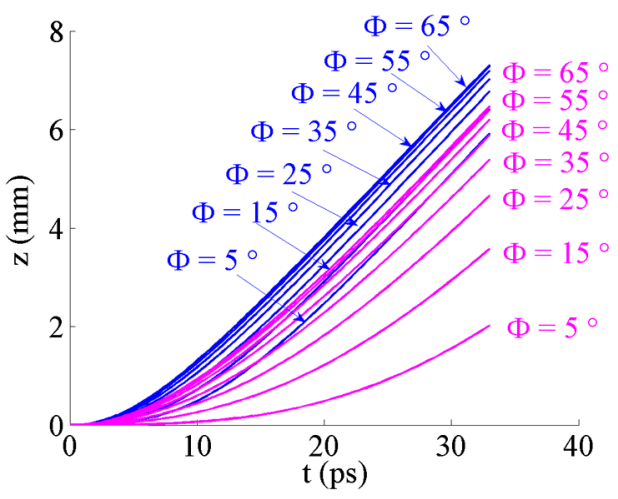

(c)

FIG. 6. (a) Evolution of $\gamma$ with time for a single electron in a $200 \mathrm{MV} / \mathrm{m} \mathrm{X}$-band gun (blue) and a $120 \mathrm{MV} / \mathrm{m} \mathrm{S}$-band gun (magenta) for different initial rf phases, (b) corresponding longitudinal electric field seen by the electron and (c) distance from cathode as a function of time. 
The electron bunch is compressed (via velocity bunching) with respect to the initial laser pulse length when running at phases near the rf zero crossing. For intermediate space charge levels, the beam in an X-band gun still behaves similarly to that in the low space charge regime, with moderate or no compression, whereas the beam in the S-band gun already behaves as that in the high space charge regime, in which no bunch compression occurs. Larger initial phases in the high space charge regime give shorter bunches in general as the freezing process is more rapid since the cathode field is closer to maximum. Accordingly, the X-band gun achieves bunch lengths which are 2 to 4 times smaller than for the S-Band gun for similar laser spot sizes and bunch charges in this regime.

The high field present in the X-band gun also allows operation at higher charges for smaller laser spot sizes [23]. The space charge limit equation, which is the direct consequence of Gauss's law, is given in Eq. (1), where $\mathrm{E}$ is the cathode field, $\mathrm{Q}$ the charge and $\mathrm{r}$ the laser spot radius. With an increase in the cathode field by a factor of two, the spot size radius can be reduced by a square root of two for the same charge and thus the initial emittance is reduced by this same factor. However, this scaling only applies for small spot sizes near the cathode surface. For larger spot sizes, nonlinear focusing effects in an X-band gun are stronger than in an S-band gun, which leads to transverse emittance degradation (i.e., the emittance does not scale with r).

$$
\mathrm{E}=\frac{\sigma}{\varepsilon_{\mathrm{o}}}=\frac{\mathrm{Q}}{\varepsilon_{\mathrm{o}} \pi \mathrm{r}^{2}}
$$

In addition, Eq. (1) is valid as long as the aspect ratio of the laser pulse is similar to that of a "pancake". For a time-elongated laser profile, i.e., "cigar shape," the space charge limit can be violated $[23,24]$. The current limit is then defined by the Child-Langmuir law with the charge proportional to the $3 / 2$ power of the product of radius times acceleration field as described in Equation (2) of [23].

\section{Beam dynamics at very low charge}

To illustrate how the final bunch length varies with the initial laser-to-rf phase and the laser spot size, simulations were performed using ASTRA [25] for the case of a $30 \mathrm{fC}$ bunch charge. These simulations were originally performed to illustrate the advantage using an X-band gun for ultrafast electron diffraction (UED) applications for which an extremely small divergence and short bunch length is desired for a charge as large as possible. As an example, for a laser spot size of $25 \mu \mathrm{m}$ in radius, which produces an initial $11 \mathrm{~nm}$ transverse emittance bunch assuming a thermal emittance of $0.9 \mathrm{~mm}$-mrad per mm laser spot size, the resulting bunch lengths exiting the X-band gun can be as small as $25 \mathrm{fs}$ rms, much shorter than $42 \mathrm{fs}$ rms, the minimum an S-band gun can produce for this laser spot

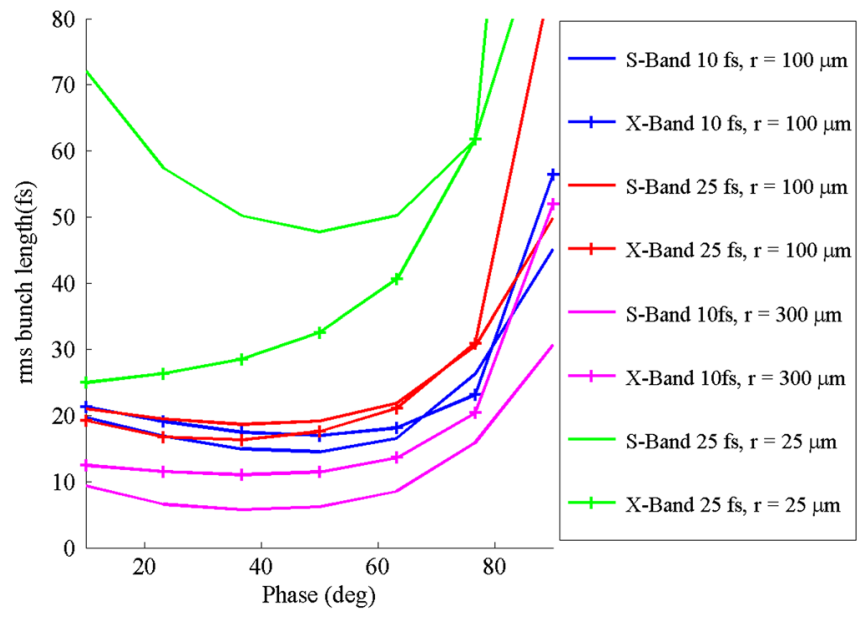

FIG. 7. RMS bunch length versus laser-to-rf phase for different laser pulse durations and spot sizes, but a fixed $30 \mathrm{fC}$ charge (i.e., $2 \times 10^{5}$ electrons) calculated at a distance $0.2 \mathrm{~m}$ from the cathode.

size. Figure 7 illustrates this and other examples-the magenta curves correspond to low space charge, the blue and red curves to moderate space charge and the green ones to high space charge.

\section{Beam dynamics at intermediate charge}

A beam parameter optimization was done for both an $\mathrm{X}$-band and S-band injector using the space charge code ASTRA for bunches of $20 \mathrm{pC}$, which was the typical bunch charge used to commission the XTA, and a typical charge used in XFELs. The X-band injector beamline was similar to that of the XTA but with the linac accelerator structure located $0.55 \mathrm{~m}$ from the cathode, instead of $0.85 \mathrm{~m}$. The S-band injector was basically the first $5 \mathrm{~m}$ of the LCLS injector which includes a 1.6 cell S-band gun operated with a $120 \mathrm{MV} / \mathrm{m}$ cathode field and a $3 \mathrm{~m}$ long linac operated at $18 \mathrm{MV} / \mathrm{m}$ and located $1.4 \mathrm{~m}$ from the cathode. Extensive simulations were run for the LCLS design and can be found in [26].

The optimizations were done in the "blow-out" regime assuming FWHM laser pulse lengths of $60 \mathrm{fs}, 120 \mathrm{fs}$ and $180 \mathrm{fs}$ (at XTA, laser pulse lengths between $60 \mathrm{fs}$ and $120 \mathrm{fs}$ were used). Both the laser-to-rf injection phase and the solenoid field amplitude were varied in an extensive 2D scan for each laser pulse length. The process was repeated for individual laser transverse spot sizes varying from a radius of $70 \mu \mathrm{m}$ to $1 \mathrm{~mm}$.

Figure 8 shows plots of the minimum emittance, the corresponding bunch length and highest peak brightness for these scans versus laser spot size radius. The peak brightness is defined as the charge in $\mathrm{pC}$ times the inverse of the product of the rms bunch length in ps and the square of the $95 \%$ transverse normalized emittance in mm-mrad. The maximum peak brightness of the X-band system is 6 times larger than that for S-band. However the spectral brightness 


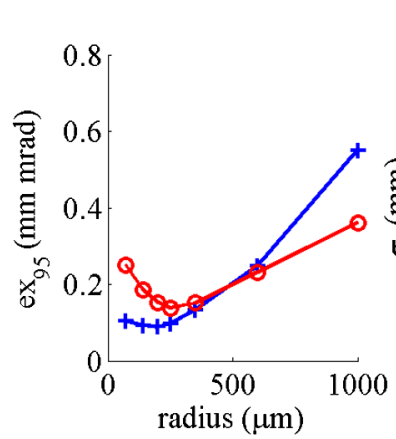

(a)

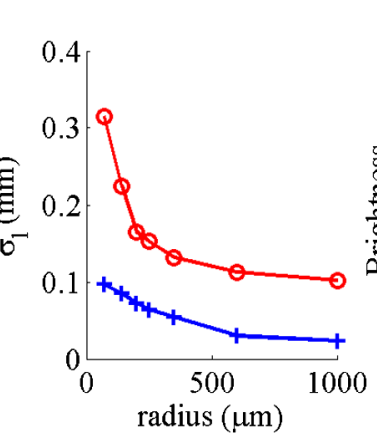

(b)

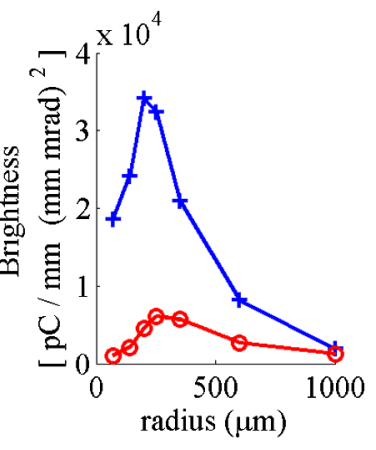

(c)

FIG. 8. Results from charge parameter scans as a function of the laser spot size radius for $20 \mathrm{pC}$ bunches where the blue crosses are for X-band (200 MV/m cathode field) and the red circles are for S-band (120 MV/m cathode field): (a) minimum $95 \%$ transverse emittance, (b) minimum bunch length, (c) maximum peak brightness.

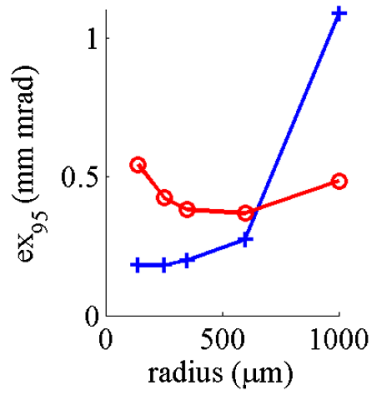

(a)

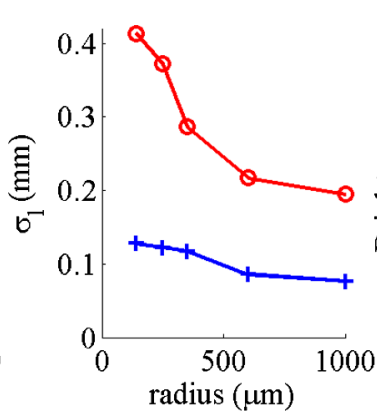

(b)

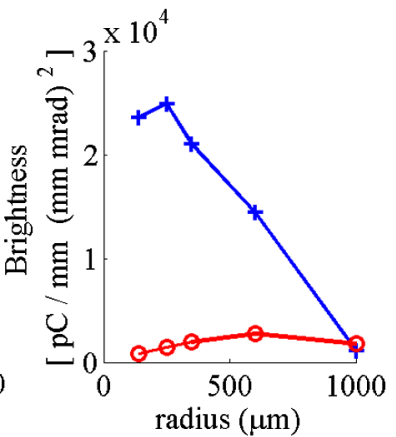

(c)

FIG. 9. Results from $100 \mathrm{pC}$ bunch charge parameter scans as a function of the laser spot size radius where the blue crosses are for X-band (200 MV/m cathode field) and the red circles are for S-band (120 MV/m cathode field \}: (a) minimum $95 \%$ transverse emittance, (b) minimum bunch length and (c) maximum peak brightness.

increase is smaller because the uncorrelated energy spread in the X-band gun is larger for a similar charge. However this is not important for FEL applications as the slice energy spread needs to be increased anyway to suppress microbunching.

\section{E. Beam dynamics at high charge}

A similar optimization was done for $100 \mathrm{pC}$ bunch charges. Again, laser pulse lengths of $60 \mathrm{fs}, 120 \mathrm{fs}$ and 180 fs FWHM were used. The results are summarized in Fig. 9. In this case, the maximum peak brightness is 10 times larger at X-band than at S-band.

Both the $20 \mathrm{pC}$ and $100 \mathrm{pC}$ cases were optimized for short laser pulses in the blow-out pancake regime, i.e., with an aspect ratio of the laser pulse length to laser transverse spot dimension close to or smaller than unity. Slightly smaller transverse emittances can be achieved with cigarshaped laser pulses as a transverse spot size smaller than the limit inferred from Eq. (1) can be generated [23]. However the reduction in emittance is very small, usually only for the core slices, and is achieved at the expense of a much longer bunch length.

\section{OPERATION OF THE X-BAND GUN AND LINAC}

\section{A. Dark current from the rf gun}

We first note that we use the term "dark current" to refer to the net electron charge from field emission that is captured/accelerated during a pulse.

It had been generally believed that a high gradient rf gun would be of limited use due to high levels of dark currents that would be coaccelerated. The Mark-0 gun produces dark currents at the level of hundreds of $\mathrm{pC}$, but we can still achieve relatively "clean," low emittance, low charge (a few pC) electron bunches. For our nominal operating conditions, the dark current is limited to a few tens of $\mathrm{pC}$ after acceleration in the linac where the $6 \mathrm{~mm}$ diameter beam tubes at each end of the $1 \mathrm{~m}$ long accelerator structure act as collimators. The transmitted dark current has a wide energy spread and transverse size, and thus can be further collimated if necessary.

The gun dark current comes primarily from the cathode and is believed to be enhanced by the surface roughness. The cathode roughness can be characterized on two distance scales. On the 1 micron scale, the roughness is 


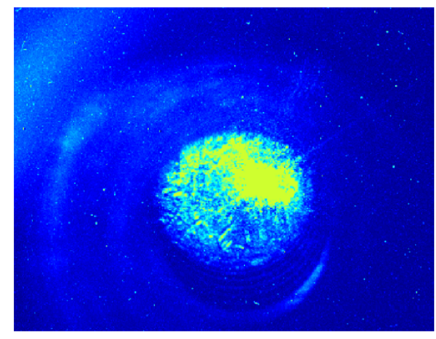

(a)

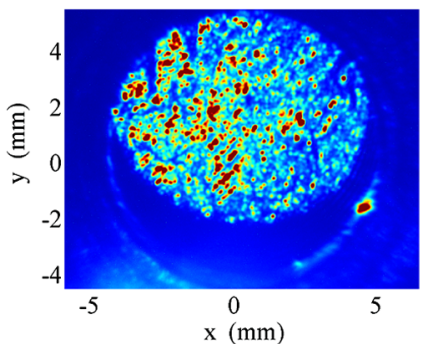

(b)

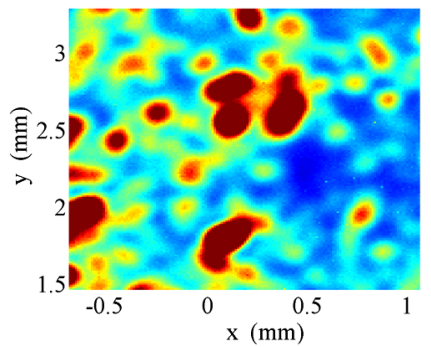

(c)

FIG. 10. Cathode surface images taken through the extraction mirror (opposite to the injection mirror) using a camera just outside of the vacuum chamber. (a) This image was taken using visible light after the gun was rf conditioned but before the first photoelectron beam was generated. The surface already appears damaged at this stage. (b) Cathode surface imaged with visible light after 2 years of operation of the gun. The center circle of bright spots fills the entire $7 \mathrm{~mm}$ diameter cathode. (c) Zoomed area of the cathode image shown in (b).

on the order of $100 \mathrm{~nm} \mathrm{rms} \mathrm{due} \mathrm{to} \mathrm{the} \mathrm{machining} \mathrm{finish}$ (unfortunately, it was not possible to diamond fly cut the cathode to reduce this). At the 10-100 micron scale, there are divots and spikes, probably 1-10 microns in height and depth, generated from rf breakdown and laser induced damage. Images of the cathode are shown in Fig. 10. The roughness is believed to contribute to the somewhat larger thermal emittance that was measured (see Sec. VI B). Also, emission and thermal emittance can vary by factors of two with grain orientation [27]. Unless the cathode is dismantled from the gun and the surface analyzed, the relative effects of roughness and grain orientation on the gun performance would be hard to evaluate. A zoomed image [Fig. 10(c)] of the illuminated cathode shows strong variations in the surface topology at a scale of typically $100 \mu \mathrm{m}$, but also down to $20 \mu \mathrm{m}$ or less.

Dark current emission is described by the FowlerNordheim equation, and electrons originating near the

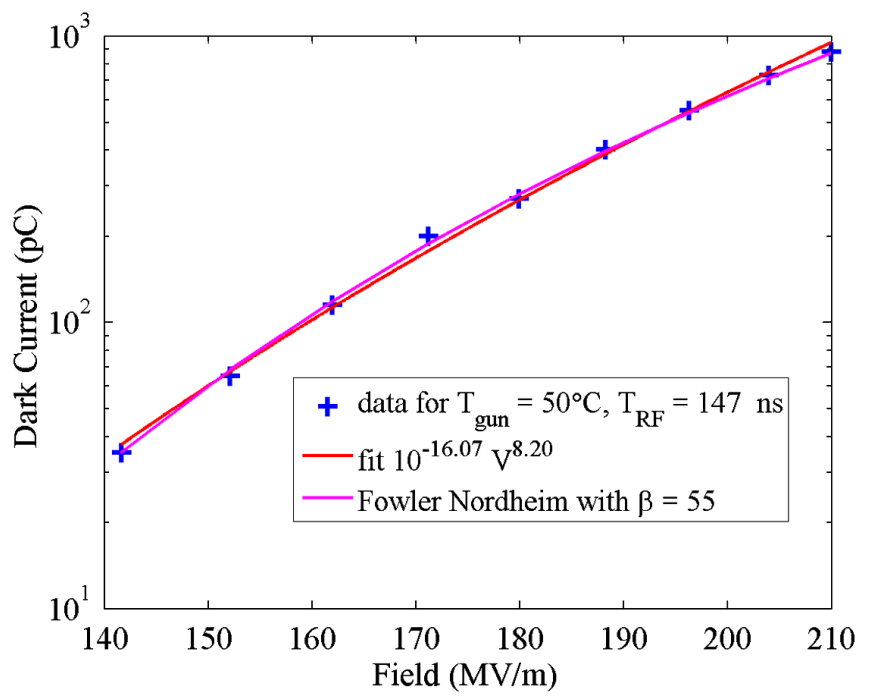

FIG. 11. Integrated dark current as a function of cathode gradient with $147 \mathrm{~ns}$ long rf pulses measured $0.56 \mathrm{~m}$ from the cathode. cathode center are readily captured in the gun. The accelerated dark current as a function of peak field on the cathode was measured with a Faraday cup located $0.56 \mathrm{~m}$ away from the cathode and is plotted in Fig. 11. The dark current energy distribution was centered at $4 \mathrm{MeV}$ for cathode gradients above $190 \mathrm{MV} / \mathrm{m}$, where we typically ran. These data were taken using $147 \mathrm{~ns}$ long, square rf input pulses, during which the cathode field reached $91 \%$ of its long-pulse limit. The gun solenoid was adjusted to maximize the captured dark current at each power level and the gun was operated a temperature of $50^{\circ} \mathrm{C}$, about $3{ }^{\circ} \mathrm{C}$ below the on-resonance value. Using a simplified form of the Fowler-Nordheim equation [28], a field enhancement factor (beta) of 55 was obtained, which is similar to beta values derived from field emission in X-band accelerators [29,30]. It should be noted, however, that the fraction of emitted electrons that are captured varies with gradient. Figure 11 shows both a power law and the FowlerNordheim equation fit to the data.

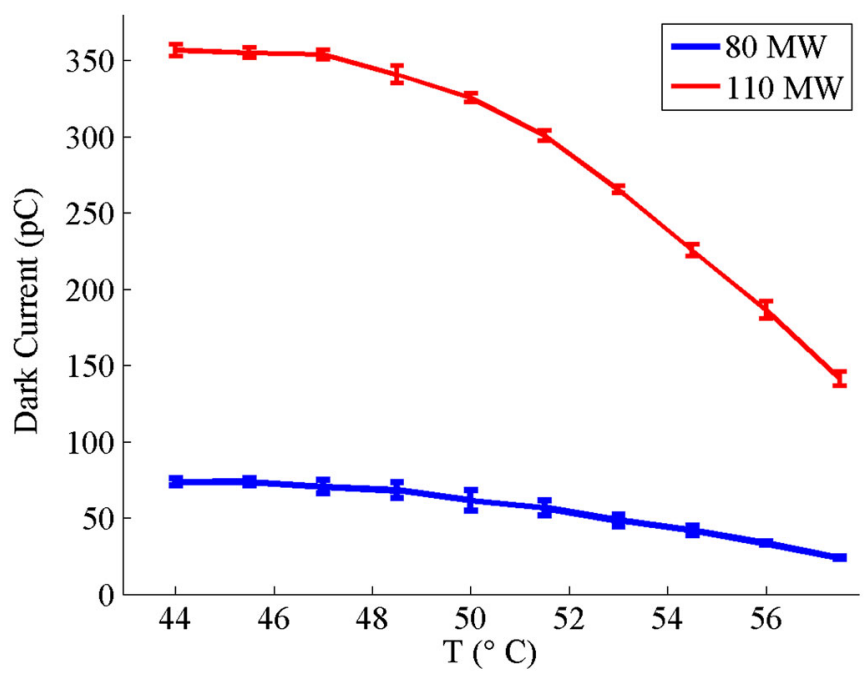

FIG. 12. Dark current as a function of gun temperature at two different SLED-II power levels with 147 ns pulses. 
The field amplitudes and phases in the 6 cells of the rf gun vary with temperature for a given input power, which changes how much dark current is produced and captured. In particular, as the temperature increases, the rf match changes and the cathode field decreases relative to the fields in the downstream cells. As a consequence, the dark current decreases, as can be seen in Fig. 12.

Not only was the cathode damaged, but the iris edges were as well, in particular when the laser energy density exceeded the ablation threshold and the laser was missteered, hitting the coupler iris. The small $(8 \mathrm{~mm})$ iris diameters of the gun made the laser setup challenging. The amount of copper removed was large enough to shift the resonant frequency by $4 \mathrm{MHz}$ (equivalent to a $19^{\circ} \mathrm{C}$ temperature change), resulting in a field profile that was no longer flat at the resonant frequency, but instead lower at the cathode end relative to the downstream end. All but the emittance data were taken before the main detuning occurred.

\section{B. Measurements of the beam energy and gun gradient}

\section{Direct energy measurement}

Figure 13 shows the expected energy of a single electron exiting the gun versus rf phase with respect to the laser arrival for various cathode field gradients. At XTA, the beam energy was measured by deflecting it just after the gun with a corrector magnet and observing the downstream change in position. We did this using both horizontal and vertical corrector magnets located $20 \mathrm{~cm}$ upstream of a Yttrium Aluminum Garnet (YAG) profile monitor, and the results are shown in Fig. 14. The energies inferred from the horizontal and vertical kicks agree well. The calibration of the correctors was accurately measured at the SLAC Magnetic Measurement Facility. The magnetic field versus current curve exhibits a

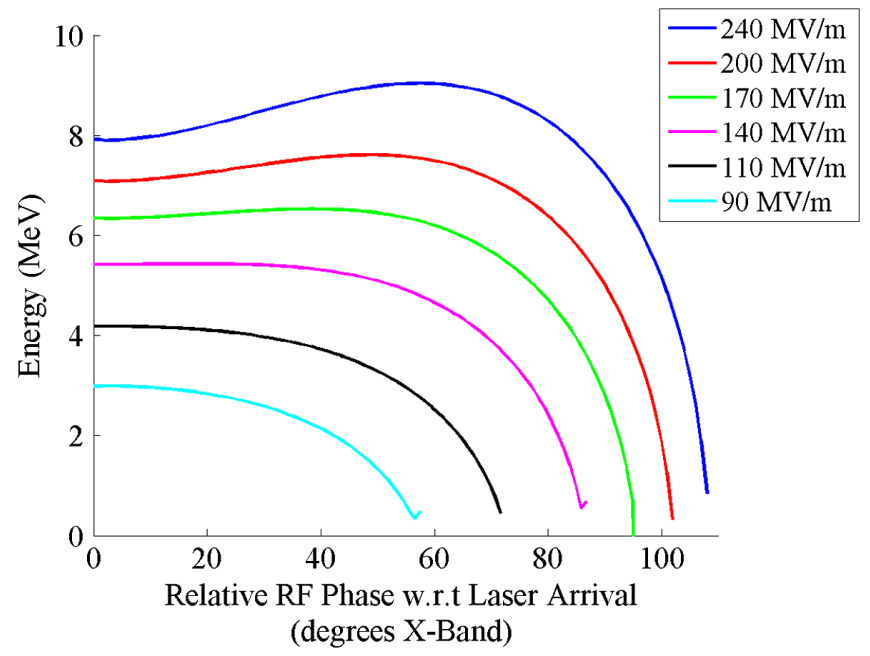

FIG. 13. Calculated single electron energy from the X-band gun versus the cathode rf phase at the laser arrival time for various cathode gradients. Zero phase corresponds to the rf zero-crossing.

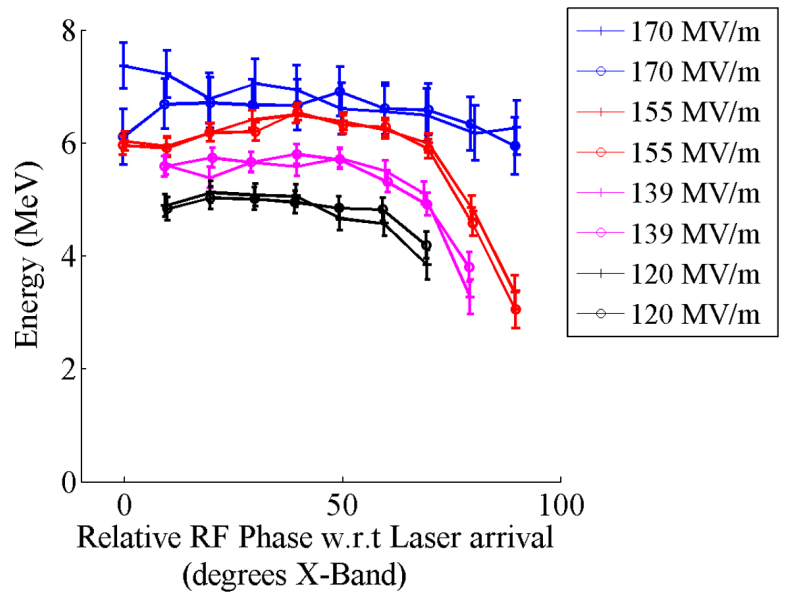

FIG. 14. Bunch energy versus rf-to-laser phase measured after the gun at different cathode gradients using either the horizontal (data points with crosses) or vertical corrector magnets (data points with circles). The gradient is based on the SLED-II power level with the expectation that $150 \mathrm{MW}$, corresponding to 15.3 MW at the gun input, yields a $190 \mathrm{MV} / \mathrm{m}$ cathode field (as discussed below).

relatively large hysteresis, with a $10 \%$ difference in slopes when cycling the magnet up or down. The agreement in the energy data for the two planes could only be obtained after accounting for this hysteresis. Note that measuring the bunch centroid at a cathode gradient of $170 \mathrm{MV} / \mathrm{m}$ was difficult as the dark current obscured the photoelectron beam, and thus the error bars on these data are larger.

The rf phase is arbitrarily chosen to be zero when the beam first appears for a $170 \mathrm{MV} / \mathrm{m}$ cathode field. For a single particle, this would correspond to the rf zerocrossing, whereas for our measurements, it is delayed as the applied field has to overcome image charge fields before the beam is accelerated. Accordingly, the phase delay is larger for the lower gradient data, and the beam

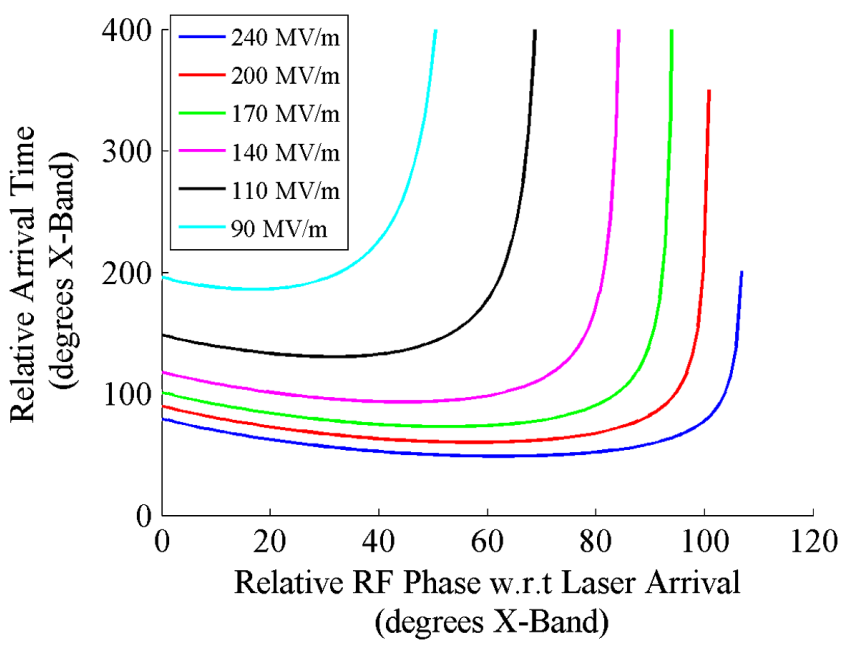

FIG. 15. Simulated RTOA versus rf phase relative to the laser arrival for different cathode gradients. 


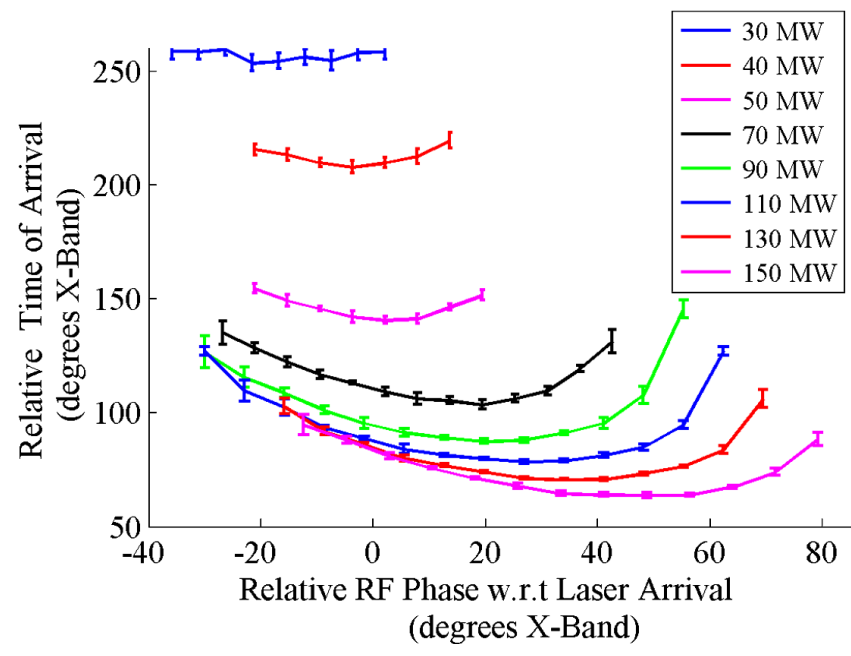

FIG. 16. Measured relative time of arrival (converted to phase at $11.424 \mathrm{GHz}$ ) versus $\mathrm{rf}$ phase relative to the laser arrival for different SLED-II power levels. The RF pulse length was $140 \mathrm{~ns}$.

turn-on occurs at a larger phase, as can be seen in Fig. 14. Given this caveat, the agreement of the data with the expectations is reasonable.

\section{Gun gradient inferred from relative time of arrival measurements}

The relative time of arrival (RTOA) of the electron bunch with respect to the $\mathrm{rf}$ phase was measured using the phase of the signal from an X-band single-cell cavity located just upstream of the accelerator structure. This monopole TM010 mode cavity has a $12.85 \mathrm{GHz}$ resonant frequency and was nominally used to provide a beam-to-rf reference phase when analyzing the dipole signals from the associated dipole cavity (i.e., as part of an rf BPM). This frequency was chosen to be offset from $11.424 \mathrm{GHz}$ so as not to "see" dark current and to allow locking to the master oscillator with "simple" rf multiply/divide steps. The signal was processed via in-phase and in-quadrature (I\&Q) analog demodulation electronics to extract phase and amplitude. Such RTOA measurements are usually performed at low

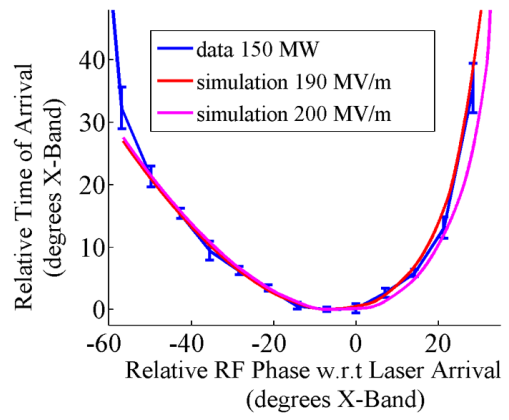

(a) energy, a few hundred $\mathrm{keV}$, to determine the electron beam energy, but not for $\mathrm{MeV}$ beams as was done here.

The beam phase was measured with respect to the master rf clock which consists of a crystal oscillator at $476 \mathrm{MHz}$. This master signal is multiplied to $2.856 \mathrm{GHz}$ and then to $11.424 \mathrm{GHz}$. The jitter of the $2.856 \mathrm{GHz}$ reference signal was measured to be $70 \mathrm{fs}$ rms. A signal source analyzer (SSA), which itself has two reference crystal oscillators, was used for this measurement. The laser oscillator was locked to this $2.856 \mathrm{GHz}$ reference signal.

The RTOA predictions and measurements are plotted in Fig. 15 and Fig. 16, respectively, and compared in Fig. 17. For the measurements, the beam-to-rf phase from the monopole cavity signal is plotted as a function of the laser-to-rf phase. This phase comparison is shown for various cathode gradients. The best fit to the $150 \mathrm{MW}$, corresponding to $15.3 \mathrm{MW}$ at the gun input, data are the $190 \mathrm{MV} / \mathrm{m}$ predictions, which is roughly the expected cathode gradient for this power (as noted above, various uncertainties make it hard to accurately predict the gradient based on the SLED-II power).

For each rf phase, 50 RTOA samples were recorded. The variation of these data are a result of the (a) laser timing jitter with respect to the rf master source, (b) rf phase jitter with respect to the $\mathrm{rf}$ master source, (c) noise in the processing electronics (down-mixing of $12.85 \mathrm{GHz}$ to reconstitute phase), and (d) fluctuations in the rf gun field amplitude. While a more detailed discussion of these sources is beyond the scope of this paper, it is worth noting that the RTOA jitter is smallest (about $0.5 \mathrm{deg} \mathrm{X}$-Band or $125 \mathrm{fs}$ - see the error bars in right-most plot in Fig. 16) at the minimum RTOA where the rf phase jitter has a negligible effect. This jitter is comparable to that of the laser timing, indicating that the other contributions are not large.

\section{Operation of the high gradient linac}

The T105 X-band traveling wave accelerator used at XTA was upgraded after a few months of operation. The newer version has input and output couplers with rounder edges at the waveguide openings-the sharp edges in the

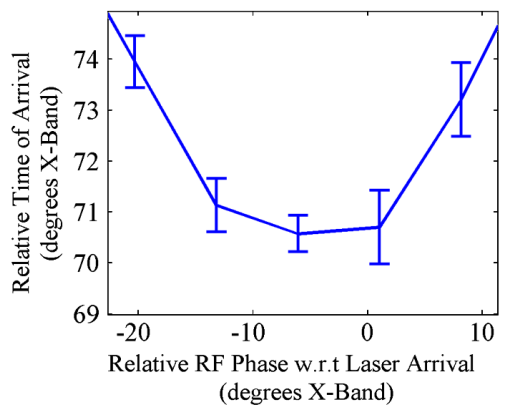

(b)

FIG. 17. (a) RTOA measurements at $150 \mathrm{MW}$ compared with simulations at gradients of $190 \mathrm{MV} / \mathrm{m}$ and $200 \mathrm{MV} / \mathrm{m}$, (b) zoom-in of the data near the RTOA minimum. RMS fluctuations were calculated using the 50 sample points. 
original version had limited its gradient to around $70 \mathrm{MV} / \mathrm{m}$ due to pulsed heating effects. The newer version is capable of running to up about $90 \mathrm{MV} / \mathrm{m}$ with a low breakdown rate $\left(<1\right.$ in $10^{6}$ pulses), and also has racetrack shaped couplers and provisions for attaching rf BPMs at each end.

The new T105 was conditioned to $80 \mathrm{MV} / \mathrm{m}$. At this gradient, no dark current ( $<$ few $\mathrm{pC}$ with $120 \mathrm{~ns}$ pulses) was measured on a Faraday Cup located $3.7 \mathrm{~m}$ downstream of it. The transverse deflector cavity (not powered at the time) was likely a factor in reducing any current-it is located at $1.5 \mathrm{~m}$ downstream of the structure and its $6 \mathrm{~mm}$ aperture acts as a collimator.

It was verified that the $1.05 \mathrm{~m}$ active-length structure achieved an $81 \mathrm{MV} / \mathrm{m}$ gradient (power-limited) using the downstream spectrometer. Most of the emittance measurements were done at beam energies of $67 \mathrm{MeV}$ and $75 \mathrm{MeV}$. A second klystron could be added to the same HV modulator for this rf station, and the power combined to increase the gradient if desired.

\section{SHORT BUNCH GENERATION}

\section{A. Simulations}

The minimum bunch length as a function of charge for a $200 \mathrm{MV} / \mathrm{m}$ cathode gradient was calculated with the multiparticle tracking codes ASTRA [25] and GPT [31]. For this study, no attempt was made to simultaneously minimize the transverse emittances.

In Fig. 18, the bunch lengths $0.2 \mathrm{~m}$ from the cathode ( $0.1 \mathrm{~m}$ downstream of the gun exit) are plotted. For each charge, the rf phase was optimized to give the minimum bunch length. The phases are small (i.e., near the zero crossing) for the low space charge regime and high for the

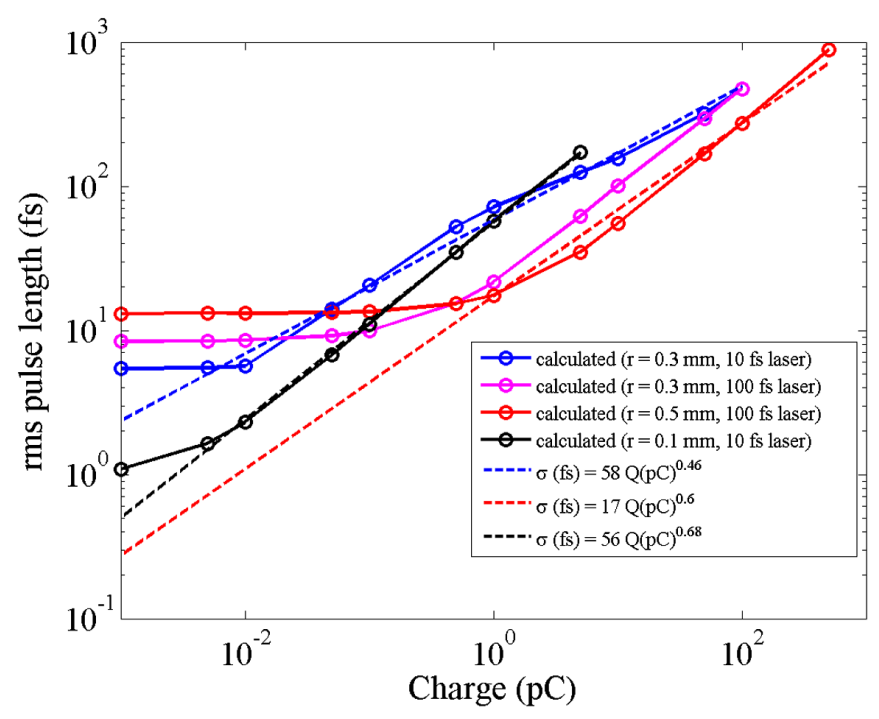

FIG. 18. Simulated bunch lengths $0.2 \mathrm{~m}$ from the cathode versus bunch charge. Also shown are power law fits to these results.
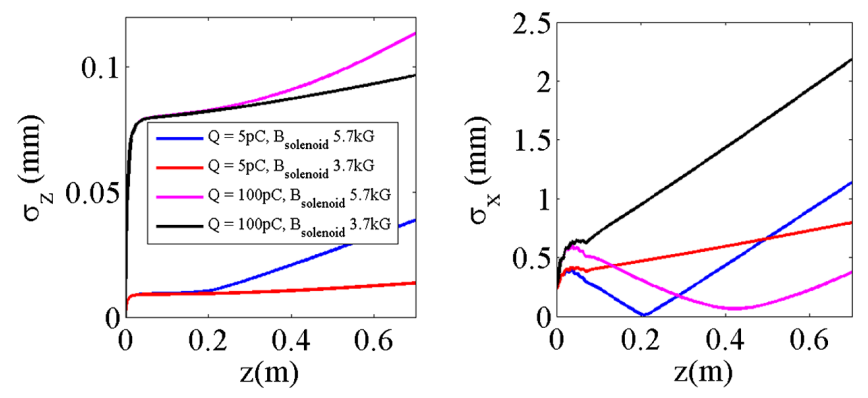

FIG. 19. Simulated bunch lengthening (a) in the drift region after the gun for different bunch charges and densities assuming a laser pulse having a $0.5 \mathrm{~mm}$ spot radius and 100 fs FWHM Gaussian longitudinal distribution. Two charge densities were simulated by changing the gun solenoid focusing strength. (b) The corresponding rms transverse beam sizes.

space charge dominated regime. The calculation was repeated for different laser pulse lengths and transverse sizes. The space charge dominated regime exhibits a strong variation of bunch length with charge as expected. Power law fits to the bunch length as a function of charge have exponents that vary from $1 / 3$ to $2 / 3$ depending on the initial laser pulse dimensions. The scaling law derived in [32] has a $1 / 3$ power dependence when the charge density stays constant, but this is rarely the case due to transverse effects as discussed earlier.

In the nonspace charge dominated regime, the electron bunch length is nearly independent of the charge and determined by the laser pulse length and the electron velocity bunching. For bunch charges less than $100 \mathrm{fC}$, the bunch length is longer for a larger laser spot size due to the radial dependence of the acceleration field as described in Eq. (2). For the same spot size, but shorter laser pulse, shorter bunch lengths are obtained due to the smaller velocity spread.

$$
\mathrm{E}_{z}(\mathrm{r}, \mathrm{z}, \mathrm{t})=\mathrm{J}_{o}(\mathrm{kr}) \mathrm{E}_{z}(\mathrm{z}, \mathrm{t})
$$

where $\mathrm{k}=2 \pi / \lambda$ and $\lambda$ is the rf wavelength.

The evolution of the bunch length and transverse size in the drift region following the gun depends on the charge density. In Ref. [33], an envelope equation is derived to calculate three-dimensional space charge effects using a linear matrix formalism. Numerical examples were studied in [34] and agree well with tracking code results.

An example of evolution of the bunch length and size, calculated with ASTRA, is shown in Fig. 19. The variation in charge density, depending on the transverse focusing, produces different lengthening rates. This is described further in [34].

\section{B. Bunch length measurements}

\section{Measurement technique}

The bunch length measurements were made using an 11cell X-Band Transverse Deflector Cavity (TCAV) [35] 


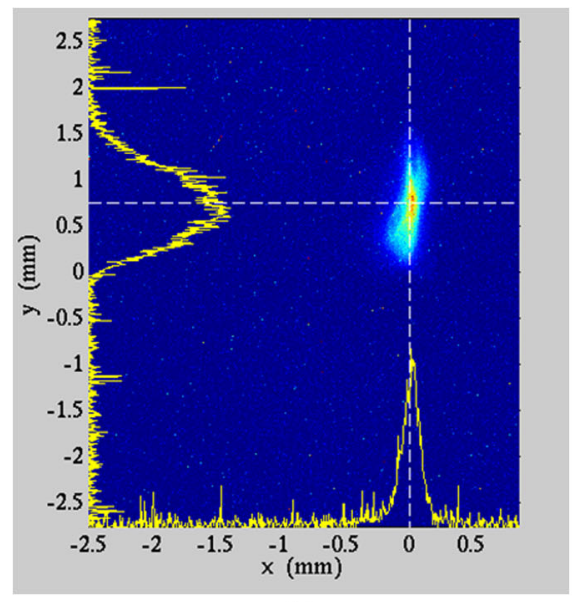

(a)

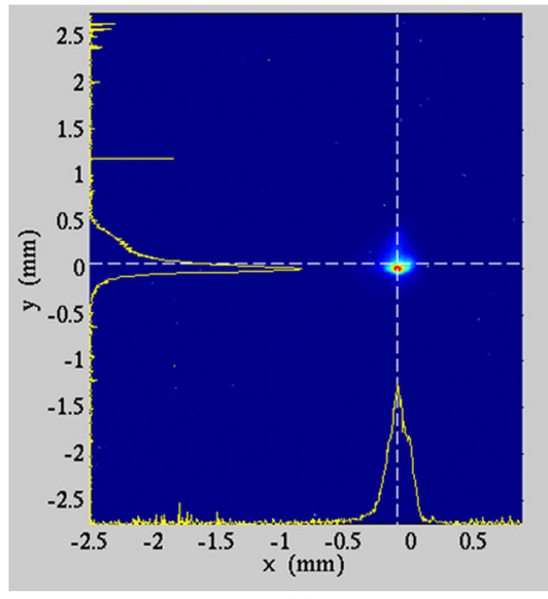

(b)

FIG. 20. (a) Streaked beam profile, and (b) beam profile with the deflector off.

that was powered by a separate rf station from that which powers the gun and accelerator. The deflector gives an angular kick in the vertical plane, which results in a vertical displacement $\Delta y$ that is a function of the position of the electrons along the bunch, $\mathrm{z}$. This displacement depends on the vertical betatron functions $\beta_{\mathrm{TCAV}}$ at the TCAV and $\beta_{\mathrm{OTR}}$ at the observation point (OTR screen in this case), and on the betatron phase advance between them, $\psi_{\text {TCAV-OTR }}$. With a cavity transverse field of $\mathrm{V}_{o}$, a bunch with momentum $p c$ and emittance that transits the cavity at the rf zero crossing is kicked differentially as:

$$
\Delta y(z)=\frac{e V o}{p c} \sqrt{\beta_{\text {Tcav }} \beta_{\text {OTR }}} \sin \psi_{\text {Tcav-OTR }}\left(\frac{2 \pi}{\lambda} z\right)
$$

and the bunch vertical size at the screen is,

$$
\begin{aligned}
\sigma_{y, \text { tot }}^{2}= & \varepsilon \beta_{\text {OTR }} \\
& +\beta_{\text {Tcav }} \beta_{\text {OTR }}\left[\frac{e V o}{p c} \sin \psi_{\text {Tcav }->\text { OTR }}\left(\frac{2 \pi}{\lambda}\right)\right]^{2} \sigma_{z}^{2} .
\end{aligned}
$$

An example of the beam profile measured with the deflector being powered is shown in Fig. 20(a), and with the power off in Fig. 20(b). The first term on the right-hand side of Eq. (4) is determined from the deflector-off bunch size, and the factor multiplying the square of the bunch length is determined by operating the cavity with the beam near the rf zero-crossing, and varying the deflector rf phase to calibrate the screen length scale in degrees of X-band. With this information and the "streaked" beam size, the bunch length is computed.

\section{Measurements with the deflector cavity}

The procedure outlined above was used to compute the bunch lengths plotted in Fig. 21. The red-cross data points were measured for an electron beam generated using a $60 \mathrm{fs}$ FWHM laser pulse and a $1 \mathrm{~mm}$ spot radius. The curves in the plot are simulations assuming the same laser duration, spot radii of $0.5 \mathrm{~mm}$ and $1 \mathrm{~mm}$, a gun solenoid field of $5.7 \mathrm{kG}$ and a cathode field of $200 \mathrm{MV} / \mathrm{m}$. The agreement is fairly good. The first series of measurements, performed in 2013 and plotted with the black crosses, were done at the early stage of the commissioning when the RF phase was drifting and the laser timing was unstable with few degree rms jitter. At that time, the laser pulse duration had not been measured accurately. Thus, it is not surprising that the agreement is better in the later measurements.

A set of measurements was also made with the goal of maximizing the peak current. The best result was $270 \mathrm{~A}$ with $290 \mathrm{pC}$ in a $460 \mathrm{fs}$ rms long bunch (one of the black

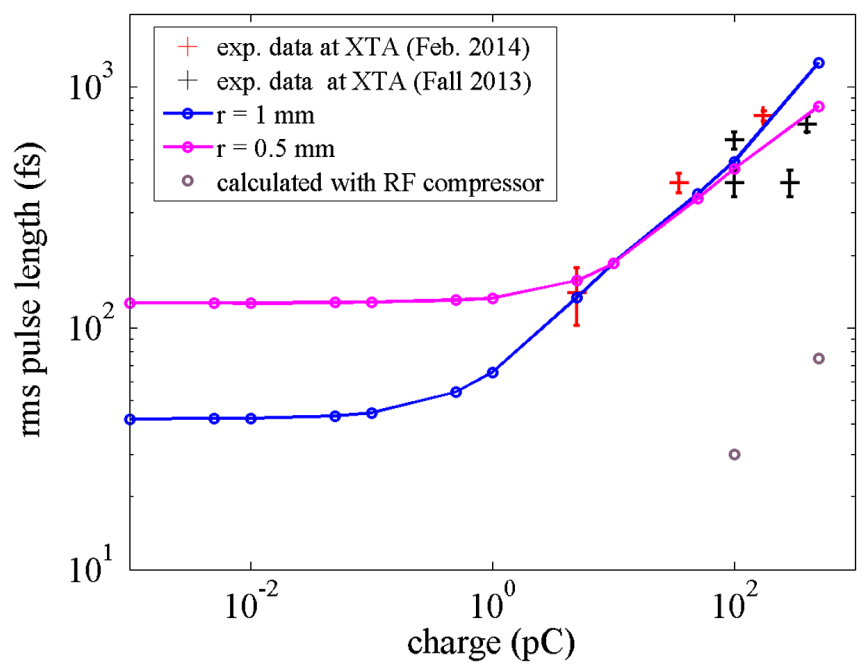

FIG. 21. Bunch lengths measured at the transverse deflector versus bunch charge, and simulated data as described in the text. Two additional simulated data points, in purple, show the bunch length when a $20-\mathrm{cm}$ long RF compressor is used. 
data points) and could be reproduced on several occasions once the linac was tuned and reasonable stability was achieved. Higher peak currents are expected for higher bunch charges. Although the RF gun has produced up to $1.2 \mathrm{nC}\left(7.2 \times 10^{9}\right.$ electrons $)$ bunches, despite a QE in the low $10^{-5}$ scale, no attempt to optimize the bunch length was made at that time.

A data point in Fig. 21 of particular interest for UED is the one with $5 \mathrm{pC}$ charge $\left(3 \times 10^{7}\right.$ electrons) where a $125 \mathrm{fs}$ rms long bunch was measured. Other injectors have achieved this performance using an S-band photocathode rf gun, but they required an additional rf accelerator for compression [36]. Our 10-cm long photocathode rf gun alone suffices to produce such short bunch lengths.

Note that the bunch length becomes "frozen" in the linac structure as close as $10 \mathrm{~cm}$ downstream of the structure entrance. Accordingly, smaller bunch lengths would be achieved if the linac was positioned closer to the cathode, for instance at $\mathrm{z}=0.6 \mathrm{~m}$. This also would produce smaller transverse emittances. In our beamline, the linac could not be located that close to the gun as space was needed for laser injection and diagnostics.

The purple circles in Fig. 21 are results from simulations to investigate how short a bunch could be achieved in a beamline similar to the XTA but with a $20 \mathrm{~cm}$ long X-band structure upstream of the linac to provide an energy chirp for velocity bunch compression. The simulations show that peak currents of up to $1.5 \mathrm{kA}$ are possible with $20 \mathrm{pC}$ bunches.

\section{Short bunch $\mathrm{THz}$ production}

With such short bunches, we expected that coherent optical transition radiation (COTR) in the $\mathrm{THz}$ regime could be generated. A $1 \mu \mathrm{m}$ thick sheet of aluminum foil

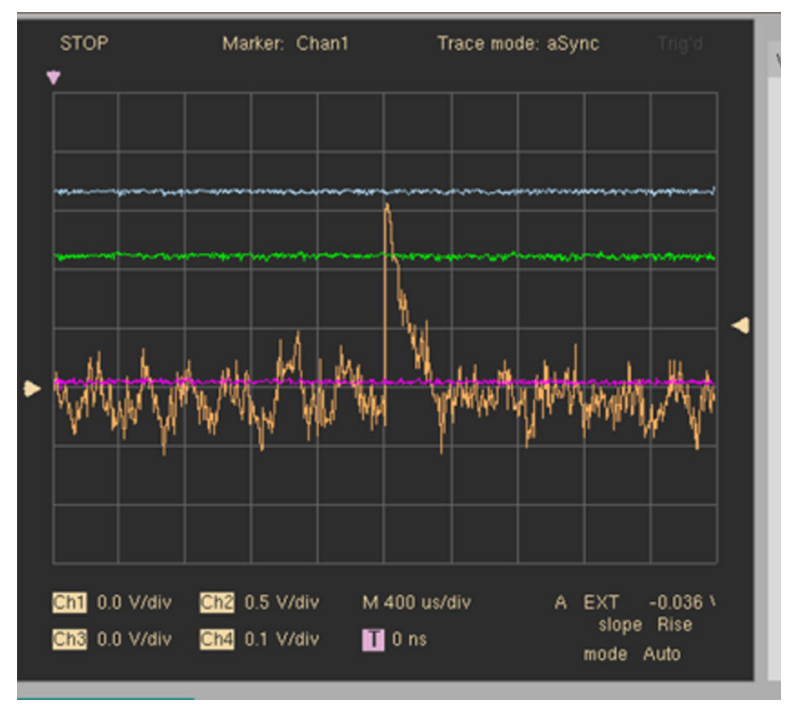

FIG. 22. Example of the signal (in orange) from the pyro-detector.

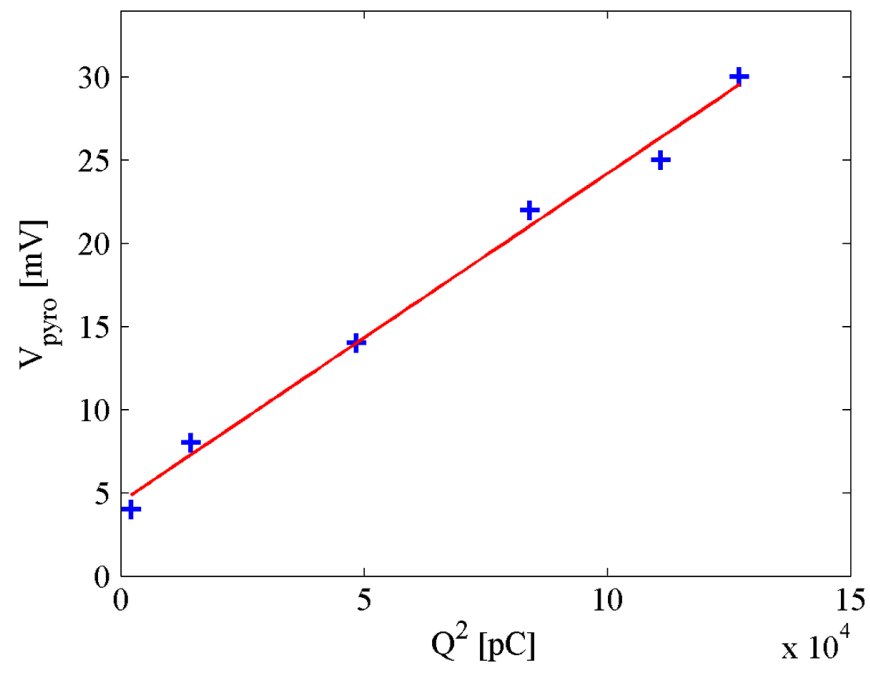

FIG. 23. Variation of the pyro-detector signal with the square of the bunch charge.

was inserted at the end of the beamline for this purpose. The light was collected in a pyro-detector and the amplitude of the signal measured to determine the total radiation energy. The COTR light propagated out of the high vacuum environment into air through a z-cut quartz window of $50 \mathrm{~cm}$ diameter, which has low attenuation and a large opening to maximize $\mathrm{THz}$ radiation collection. The light was then guided using a $6 \mathrm{~cm}$ long copper cone before being collected into a pyro-detector that was located approximately $15 \mathrm{~cm}$ from the source. The pyro-detector was calibrated using IR light and produced a $33 \mathrm{mV}$ signal per $\mu \mathrm{J}$ of light without any additional amplification. For a bunch charge of $300 \mathrm{pC}, 2 \mu \mathrm{J}$ of energy was collected as shown in Fig. 22. This value is consistent with a pulse length of $400 \mathrm{fs}$ rms and transverse source dimensions of $100 \mu \mathrm{m} \mathrm{rms}$ in both planes-the predicted radiation spectrum is peaked at $0.2 \mathrm{THz}$.

When transition radiation is emitted coherently, the energy is proportional to the square of the number of particles. By varying the radius of the laser spot, which varies the bunch charge, we verified that the collected energy is linear with the square of the charge, as shown in Fig. 23.

\section{TRANSVERSE EMITTANCE MEASUREMENTS}

\section{A. Emittance measurement setup and emittance optimization}

Transverse emittances were measured downstream of the accelerator structure by varying the strength of a quadrupole magnet while recording the profile of the light produced from an optical transition radiation (OTR) screen located $1.6 \mathrm{~m}$ downstream of the quadrupole magnet. The resolution of the system was limited by the camera lens and is estimated to be $15 \mu \mathrm{m} \mathrm{rms}$ using a standard resolution test pattern known as a United States Air Force (USAF) 


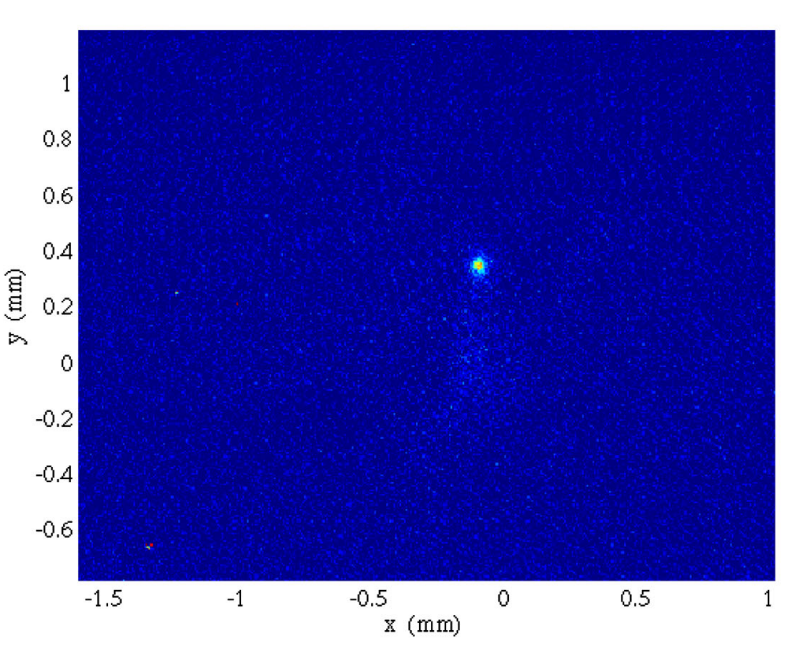

(a)
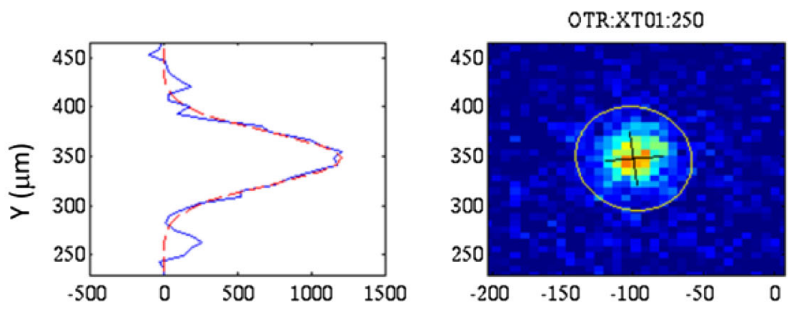

$$
\begin{aligned}
& <x>=-99.6 \mu \mathrm{m} \\
& <y>=347.7 \mu \mathrm{m} \\
& \sigma_{x}=20.7 \mu \mathrm{m} \\
& \sigma_{y}=26.3 \mu \mathrm{m}
\end{aligned}
$$

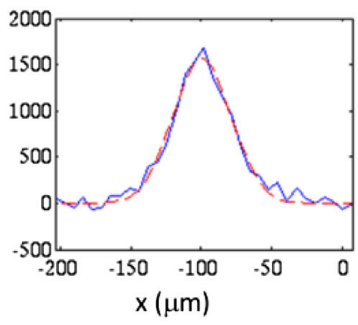

(b)

FIG. 24. Electron beam profile on the OTR screen for a bunch with low charge: (a) image on the screen and (b) Gaussian fits.

target. However, this resolution was not measured in-situ with the USAF target replacing the screen, but on an adjacent setup where we tried to reproduce the same OTR imaging configuration as that on the beamline, i.e., same aperture and macrolens focusing. In this configuration, our best resolution was $15 \mu \mathrm{m}$ rms defined such that a $50 \%$ contrast was visible while imaging the 64 line pairs of the USAF target. The OTR imaging resolution of the electron beam was no better and was likely worse.

With a 45 degree orientation of the screen in the horizontal plane relative to the beam axis, the depth of focus was small as the macrolens had a large aperture to collect as much light as possible. To measure the beam in the focal plane, the electron beam was steered horizontally until a minimum beam size was measured. An image of the electron beam is shown in Fig. 24 for a small beam size, for which Gaussian fits give rms values of $21 \mu \mathrm{m}$ and $26 \mu \mathrm{m}$ in the horizontal and vertical planes, respectively.

Emittances were typically optimized and measured at beam energies from $65 \mathrm{MeV}$ to $70 \mathrm{MeV}$. For the bunch sizes measured, the space charge effects from focusing the bunches were negligible. The optimization procedure to reach the minimum emittance consisted of steering the laser to position it at the cathode center, or to a "sweet spot", then steering the beam through the linac and minimizing the bunch energy spread. The emittance was then measured for various solenoid values. This was then repeated for different rf-to-laser phases. We did not attempt to minimize the bunch length, but they were fairly short as 50 fs FWHM laser pulses were used.

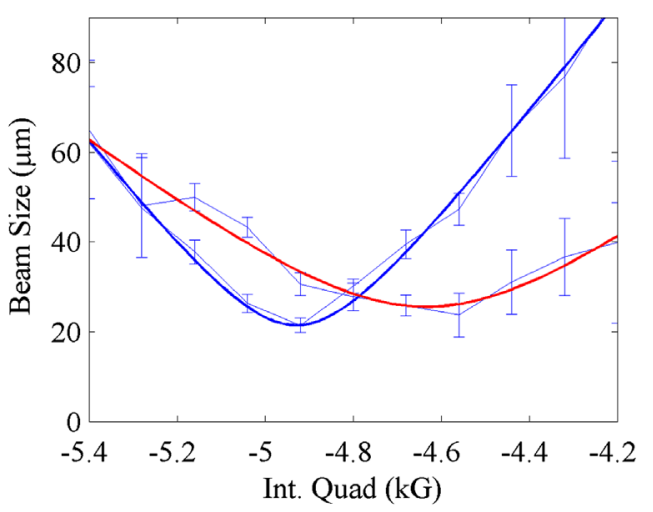

(a)

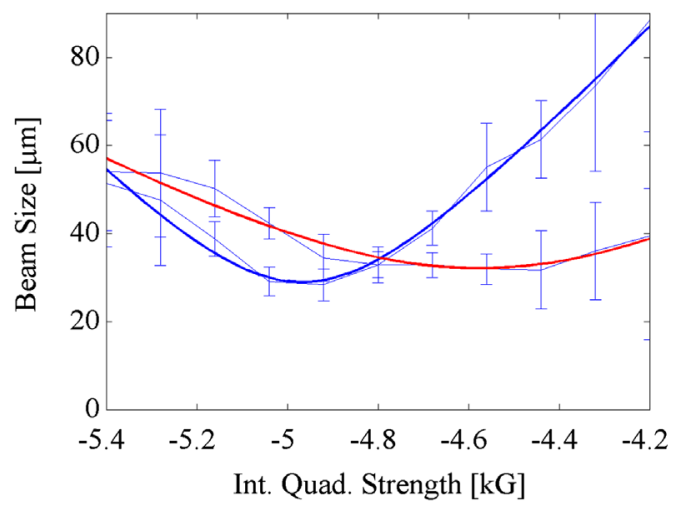

(b)

FIG. 25. Beam size versus quadrupole strength: (a) rms beam size obtained from Gaussian fits, (b) rms beam size obtained using a 5\% area cut. The blue (red) curves are fits to the horizontal (vertical) data. The rms emittances are $0.32 \mathrm{~mm}-\mathrm{mrad}$ and $0.28 \mathrm{~mm}-\mathrm{mrad}$, respectively, in the horizontal and vertical plane for the rms fits. They are $0.36 \mathrm{~mm}$-mrad and $0.28 \mathrm{~mm}$ - $\mathrm{mrad}$, respectively, in the horizontal and vertical plane for the Gaussian fits. 
The best emittance values measured after optimization and resolution corrections were $0.22 \mathrm{~mm}-\mathrm{mrad}$ at $5 \mathrm{pC}$, $0.43 \mathrm{~mm}$-mrad at $30 \mathrm{pC}$ and $0.66 \mathrm{~mm}-\mathrm{mrad}$ at $100 \mathrm{pC}$. All these values were reproducible but unfortunately they were obtained after the gun had already been damaged. The cathode emission was very nonuniform (see Sec. VI E), which made the optimization as a function of charge tedious. Indeed, when opening the iris that defines the laser spot size on the cathode, the centroid would move as well, and the thermal emittance would change (due to the change in surface topology), requiring further tuning. Below, we present the results for a few specific charges for which the laser spot was scanned around the center of the cathode to find a "sweet spot," giving the smallest emittance.

\section{B. Emittance at low charge}

A careful optimization of emittance was done at low charge $(5 \mathrm{pC})$ for a $1 \mathrm{~mm}$ diameter laser spot $(0.25 \mathrm{~mm} \mathrm{rms}$ width) on the cathode. The horizontal and vertical emittances, respectively, were $0.32 / 0.28$ from a Gaussian fit, and $0.36 / 0.28 \mathrm{~mm}$-mrad from the rms when the profiles were truncated to exclude $5 \%$ of the integrated signal located in the tails (i.e., a "5\% area cut")—-the scans are shown in Fig. 25. We then calculated the emittance after subtracting in quadrature the imaging system resolution from the beam size for different possible values of resolution. The results are summarized in Fig. 26. In particular, the $0.28 \mathrm{~mm}$-mrad emittance reduces to $0.22 \mathrm{~mm}$-mrad when an imaging resolution of $15 \mu \mathrm{m}$ is assumed, which we take as the "best" emittance value. For other locations of the laser spot, the emittance was up to three times larger. Given our operating parameters, the electron bunch length was calculated to be $125 \mathrm{fs}$ rms.

With the low bunch charge, it is reasonable to assume our measurements represent the thermal emittance, which when normalized by the rms laser spot size, yields $0.9 \mathrm{~mm}$-mrad per mm rms (using our best, resolution corrected value).

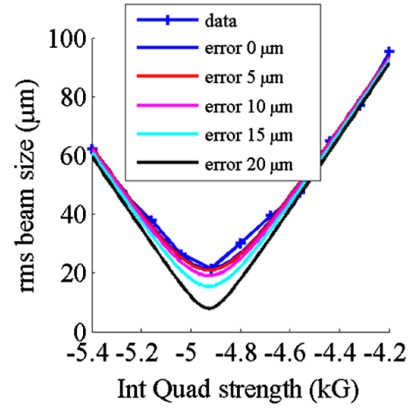

(a)

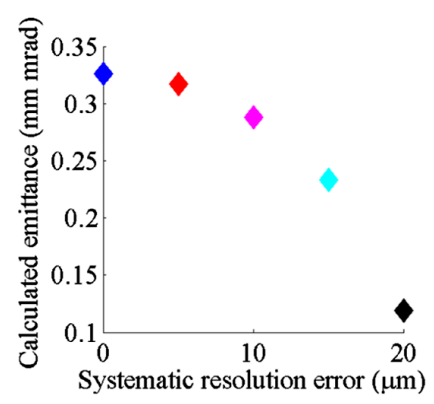

(b)
FIG. 26. Emittance calculated from the horizontal data (rms with area cut) shown in Fig. 28. after deconvolving the optics system resolution: (a) beam size versus quadrupole strength fits with the data corrected for various resolution contributions and (b) the resulting emittances versus the resolution.

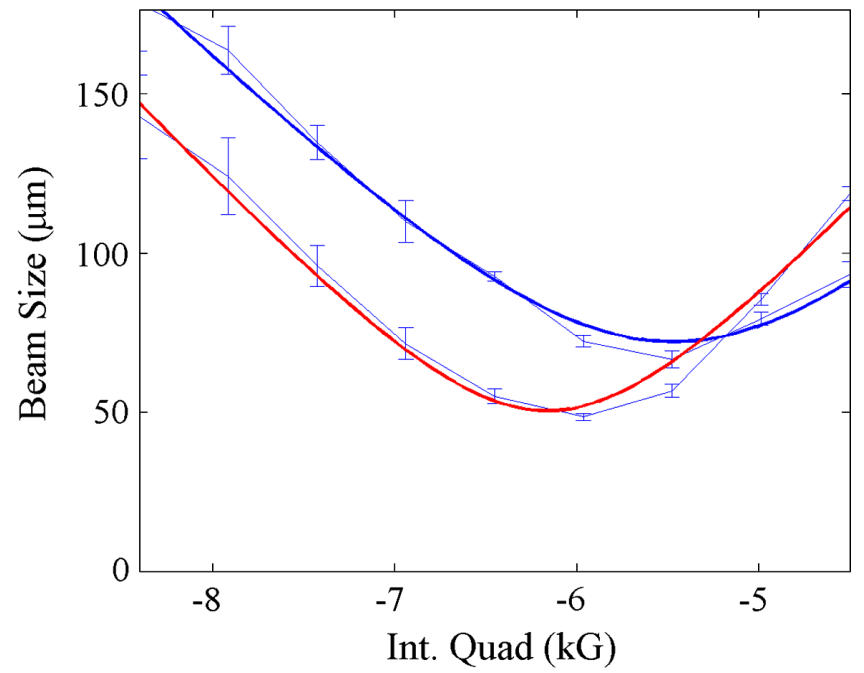

FIG. 27. Quadrupole scans with $30 \mathrm{pC}$ bunch charge: (blue) horizontal plane and (red) vertical plane. The fits give a normalized horizontal emittance of $0.47 \mathrm{~mm}-\mathrm{mrad}$ and a normalized vertical emittance of $0.43 \mathrm{~mm}-\mathrm{mrad}$.

This is in the range of values measured with S-band guns, for example, 0.6 [37] and $1.0 \mathrm{~mm}-\mathrm{mrad}$ per $\mathrm{mm} \mathrm{rms}$ [38]. The LCLS gun thermal emittance is $0.9 \mathrm{~mm}$-mrad per $\mathrm{mm}$ rms [39]. Note our measurements were done with a photoemission field of $122 \mathrm{MV} / \mathrm{m}$ (40 degrees from the zero crossing of a $190 \mathrm{MV} / \mathrm{m}$ peak field), much higher than $60 \mathrm{MV} / \mathrm{m}$ (30 degrees from the zero crossing of a $120 \mathrm{MV} / \mathrm{m}$ peak field) at which S-band gun thermal emittances are usually measured. Photoemission theory, as described for instance in [40], suggests that a higher thermal emittance is expected at a higher field, but we did not observe this. Note that our laser wavelength was $266 \mathrm{~nm}$, which should be more favorable for low emittance than the $253 \mathrm{~nm}$ wavelength used for the LCLS gun.

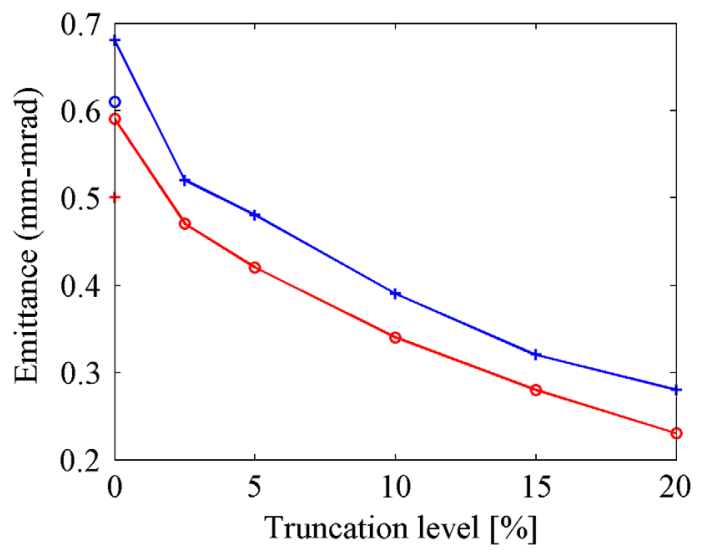

FIG. 28. Emittances computed from the $30 \mathrm{pC}$ data as a function of the truncation level used in computing the rms bunch sizes. The Gaussian fit results are plotted at $0 \%$ truncation. The blue points are from the horizontal scans and the red points are from the vertical scans. 


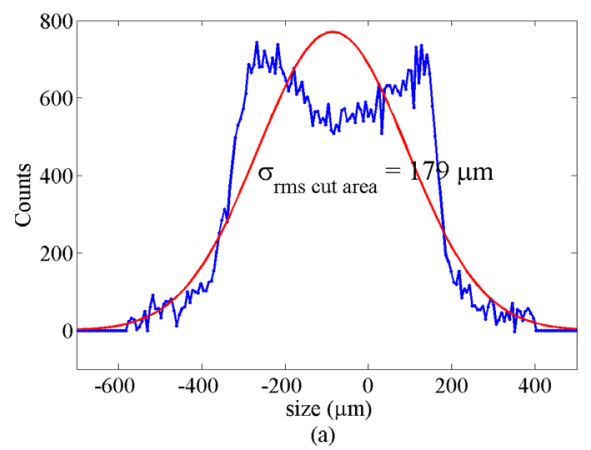

Horizontal beam profile at point (a) in plot (c).

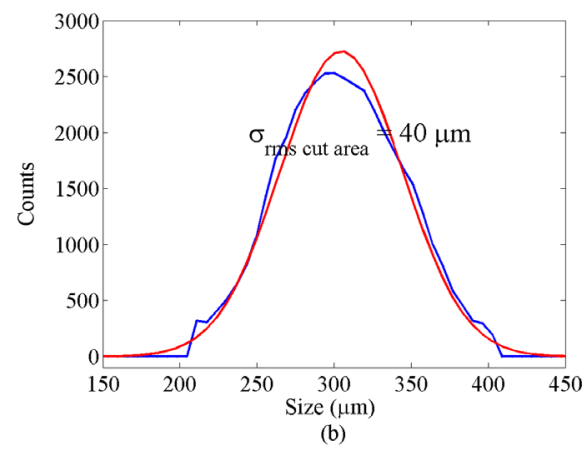

Vertical beam profile at point (b) in plot (c).

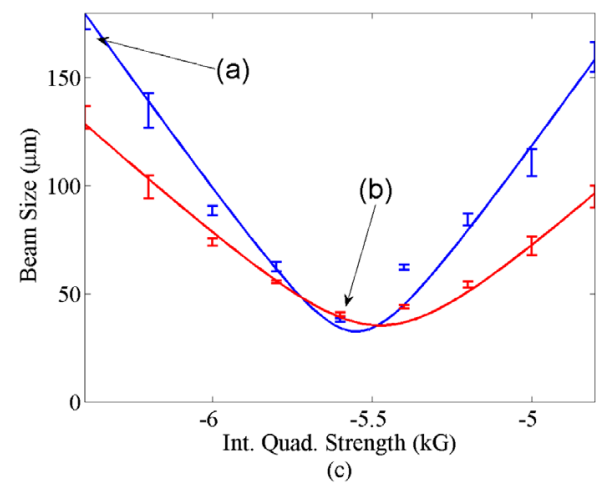

Quadrupole scans (blue is horizontal and red is vertical).

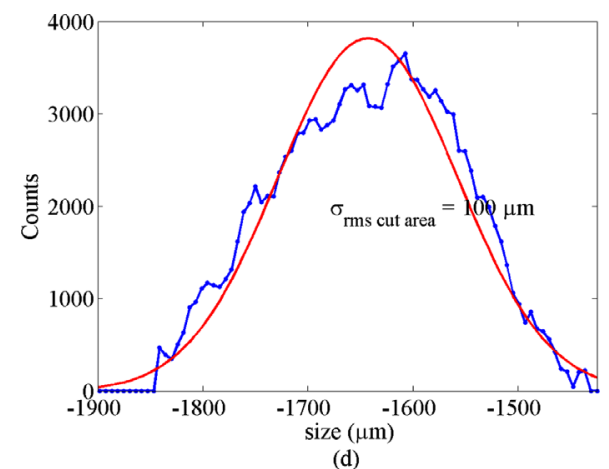

Vertical beam profile at point (d) in plot (f).

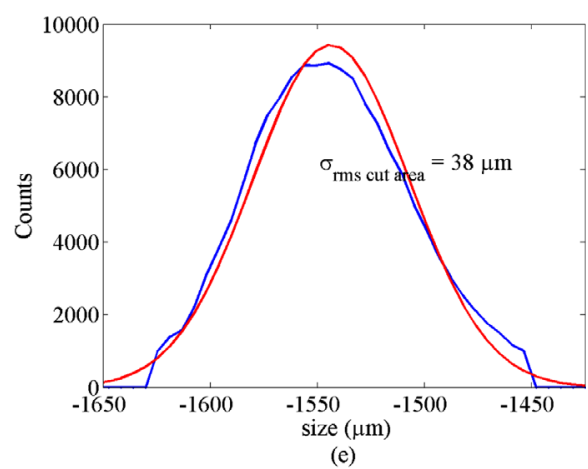

Vertical beam profile at point (e) in plot (f).

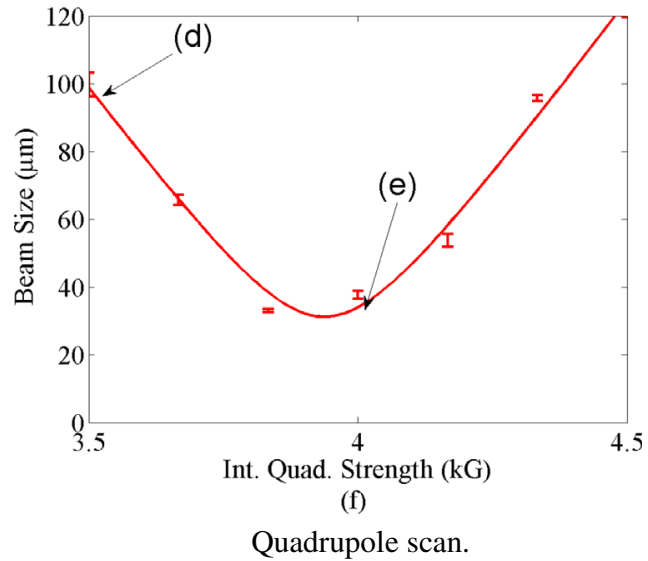

FIG. 29. Quadrupole scans and examples of bunch profiles for $100 \mathrm{pC}$ bunches. The resulting normalized emittances are $1.09 \mathrm{~mm}$-mrad and $0.81 \mathrm{~mm}$-mrad, respectively, from the fits to the horizontal data in plot (c), and the vertical emittance is $0.66 \mathrm{~mm}$-mrad from the fit to the data in plot (f). 
A preliminary study of photoemission using $400 \mathrm{~nm}$ light (second harmonic of the IR laser) instead of $266 \mathrm{~nm}$ light (third harmonic) was done at low charge. Bunches shorter than $200 \mathrm{fs}$ rms were achieved and the details are reported in [41]. Somewhat smaller emittances were obtained: $0.25 \mathrm{~mm}$-mrad in both planes for $7 \mathrm{pC}$ bunches. These values were computed from the rms bunch sizes (5\% area cut) without deconvolving the optical system resolution.

\section{Emittance at intermediate charge}

The best emittance achieved with $30 \mathrm{pC}$ bunches was $0.43 \mathrm{~mm}$-mrad (based on rms beam sizes with a 5\% area cut) using a $300 \mu \mathrm{m}$ laser spot size radius (see Fig. 27). This is a factor of two worse than the best value obtained in simulations for the present XTA geometry, i.e., with the linac located at $0.85 \mathrm{~m}$ from the cathode. For an ideal machine assuming a thermal emittance of $0.9 \mathrm{~mm}$-mrad per $\mathrm{mm}$ rms laser spot size, a $160 \mu \mathrm{m}$ laser spot size radius, a linac located $0.55 \mathrm{~m}$ from the cathode and optimal laser shaping, the best simulated emittance $\left(\varepsilon_{100 \%}\right)$ is $0.1 \mathrm{~mm}$-mrad. Given the low QE of our cathode, we were unable to produce enough charge to get measurable OTR signals for this laser radius.

For those interested in a measure of the central slice emittance of the bunches (e.g., for lasing), Fig. 28 is a plot of the emittance versus various levels of "tail" truncation.

Emittances of $20 \mathrm{pC}$ bunches were also measured. They were typically $0.35 \mathrm{~mm}$-mrad, about twice that achieved in LCLS.

\section{Emittance at high charge}

For $100 \mathrm{pC}$ bunch charges, the measured emittances were typically around $0.8 \mathrm{~mm}$-mrad (based on rms beam sizes with a 5\% area cut). Figure 29 shows examples of the

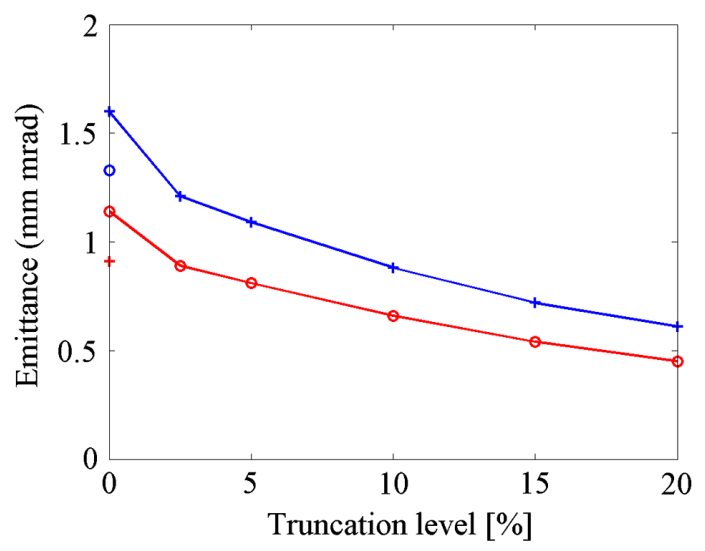

FIG. 30. Emittance analysis of the 100 pC data in Fig. 29(c) as a function of truncation level used in computing the rms bunch sizes. The Gaussian fit results are plotted at $0 \%$ truncation. The blue points are from the horizontal scans and the red points are from the vertical scans. measured beam profiles and emittance scans. In Fig. 29(a), the horizontal beam profile exhibits a double bump and the measured beam emittance was $1.09 \mathrm{~mm}$-mrad. This shape resulted from having two strong photoemission areas. The vertical beam profile shown in Fig. 29(b) is a more typical example, where the emittance is $0.81 \mathrm{~mm}$ mrad. Some vertical measurements yielded emittances as low as 0.66 mm-mrad [Figs. 29(d), (e), and (f)], but were not easily reproduced. Figure 30 shows the emittances computed at various levels of "tail" truncation.

At this charge level, most of the measured horizontal emittances were worse than the vertical ones, independent of the laser spot location. This may be wakefield related, in particular, the injection and extraction mirrors were located $0.55 \mathrm{~m}$ from the cathode and the aperture was limited to $6 \mathrm{~mm}$ in the horizontal plane, but $25 \mathrm{~mm}$ in the vertical plane.

\section{E. Emittance degradation}

The terms contributing to the emittance can be grouped in three categories as expressed in Eq. (5).

$$
\varepsilon=\sqrt{\left(\mathrm{r} \varepsilon_{\text {thermal }}\right)^{2}+\varepsilon_{\text {mismatch }}^{2}+\varepsilon_{\text {angular momentum }}^{2}}
$$

The first term is the intrinsic emittance, which is the rms laser spot size times the normalized thermal emittance. This is basically the minimum emittance that can be achieved. The thermal emittance depends on the work function of the cathode material, on the wavelength of the laser, on the orientation of the copper grains [27] and possibly on the polarization of the laser for non-normal incidence on the cathode.

The second term includes all of the nonlinear terms which cannot be corrected by emittance compensation, which is a linear process. It includes nonlinear effects from chromaticity, the curvature of the rf fields and nonuniform charge density-for example, see [42]. Simulations show that for a $60 \mathrm{fs}$ laser pulse with a $0.5 \mathrm{~mm}$ radius and a $20 \mathrm{pC}$ bunch of zero initial emittance, the bunch emittance would grow less than $0.1 \mathrm{~mm}$-mrad with optimal emittance compensation.

The last term comes from the angular momentum acquired at emission if the magnetic field is nonzero at the cathode. It is described by Eq. (6), and is based on the conservation of angular momentum.

$$
\varepsilon_{\text {angular momentum }}[\mathrm{mm}-\mathrm{mrad}]=0.3 \mathrm{~B}_{\mathrm{z}}[\mathrm{mT}] \sigma_{\mathrm{r}}^{2}[\mathrm{~mm}]
$$

where $\mathrm{B}_{z}$ is the longitudinal magnetic field on the cathode and $\sigma_{r}$ is the rms laser radius.

For a $1.5 \mathrm{~mm}$ diameter laser spot size and a $140 \mathrm{G}$ longitudinal field at the cathode, this emittance contribution would be comparable to the thermal emittance assuming a $0.9 \mathrm{~mm}$-mrad per $\mathrm{mm}$ rms laser spot size. Such a field 


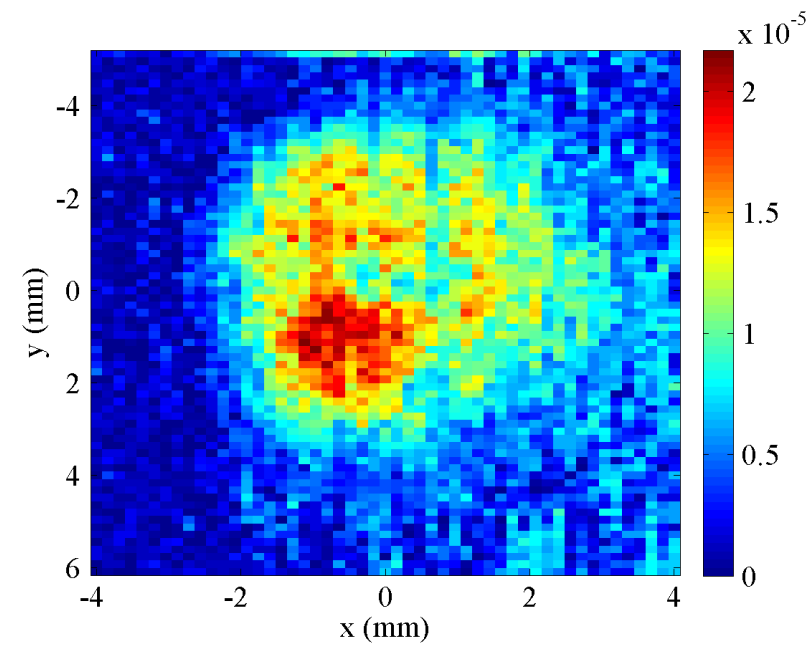

FIG. 31. Typical QE map of the cathode. The length scale at the cathode was determined from the mirror actuator calibration and its distance from the cathode.

would present if our solenoid is misaligned by $900 \mu \mathrm{m}$ longitudinally when operating at the nominal field of $5.7 \mathrm{kG}$. We believe that the null position of the solenoid field is aligned to the cathode much better than this, so this emittance growth contribution is likely negligible.

Given that a reasonable thermal emittance was achieved with $1 \mathrm{~mm}$ diameter laser spots despite the roughness of the cathode surface, the emittance growth at higher charge is likely from the nonuniformity of the photoemission, which degrades the effectiveness of the emittance compensation. Figure 31 shows an example of the QE variation over the surface. The emittances at higher charge were up to three times larger depending on where the laser was positioned. Unfortunately, we have not had the opportunity to try the new Mark-1 gun where the cathode is still pristine.

\section{BEAM POSITION AND ENERGY STABILITY}

The stability of the electron beam was mainly determined by the amplitude jitter of the rf that powers the XTA. The rf phase jitter, laser arrival time jitter and laser pointing stability have lesser effects on the beam stability. The fractional $\mathrm{rf}$ field amplitude variation (rms) is typically a few $10^{-3}$, but lower values, down to $10^{-4}$, have been measured, especially at night. Measurements of typical phase and amplitude jitter, sampled over a small

TABLE I. rf phase and amplitude jitter measurements.

\begin{tabular}{lcc}
\hline \hline Signal/Stability & $\begin{array}{c}\text { RMS phase } \\
\text { (degrees X-band) }\end{array}$ & RMS field variation \\
\hline LLRF drive & $0.2^{\circ}$ & $2.9 \times 10^{-3}$ \\
TWT klystron drive & $0.3^{\circ}$ & $7.2 \times 10^{-3}$ \\
SLED-II output & $0.25^{\circ}$ & $1.9 \times 10^{-3}$ \\
\hline \hline
\end{tabular}

fraction of the pulse length, are listed in Table I for different stages of the rf amplification chain (the klystron runs near saturation so the travelling wave tube (TWT) drive variations are suppressed).

The laser is transported along a $37 \mathrm{~m}$ transfer line from the laser room to the accelerator. The transport optics consists of a series of lenses and mirrors which perform relay-imaging of the $4 \mathrm{~mJ}$ IR laser pulse. Despite the very long transport distance and thanks to the relay-imaging system, a very good pointing stability has been achieved with shot-to-shot fluctuations on the virtual cathode that are less than a few $\%$ of the rms spot size.

\section{A. Transverse beam position and size stability}

To quantify the stability, we recorded the electron beam position and size in a series of 500 consecutive pulses measured at $2 \mathrm{~Hz}$ on the OTR beam profile monitor located $6 \mathrm{~m}$ from the gun cathode. The data were taken with $30 \mathrm{pC}$ bunches and the results are shown in Fig. 32.

The rms centroid jitter was $12.4 \%$ and $14.4 \%$ of the rms beam size in the horizontal and vertical planes, respectively.
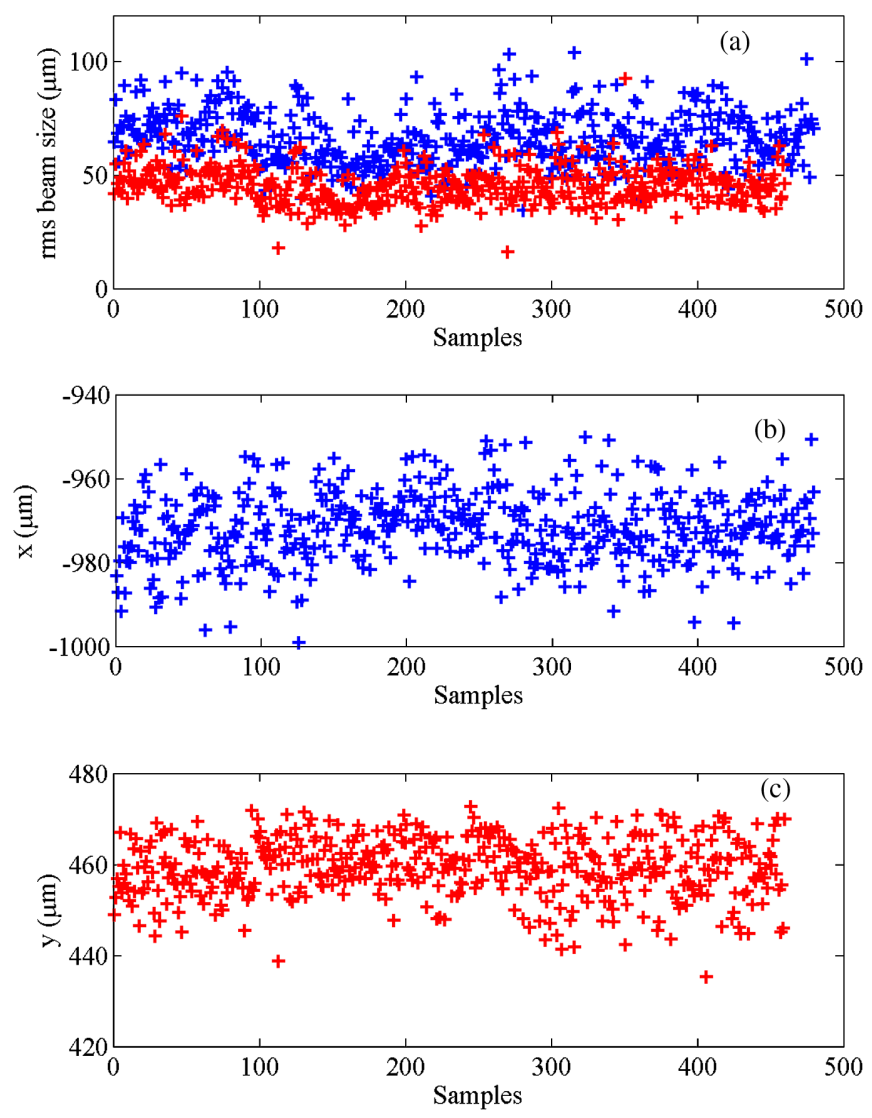

FIG. 32. Transverse stability of the electron beam measured on 500 consecutive shots (during a 50 second period with a $10 \mathrm{~Hz}$ pulse rate). (a) Vertical, in red, and horizontal, in blue, rms beam sizes, (b) horizontal position and (c) vertical position. 

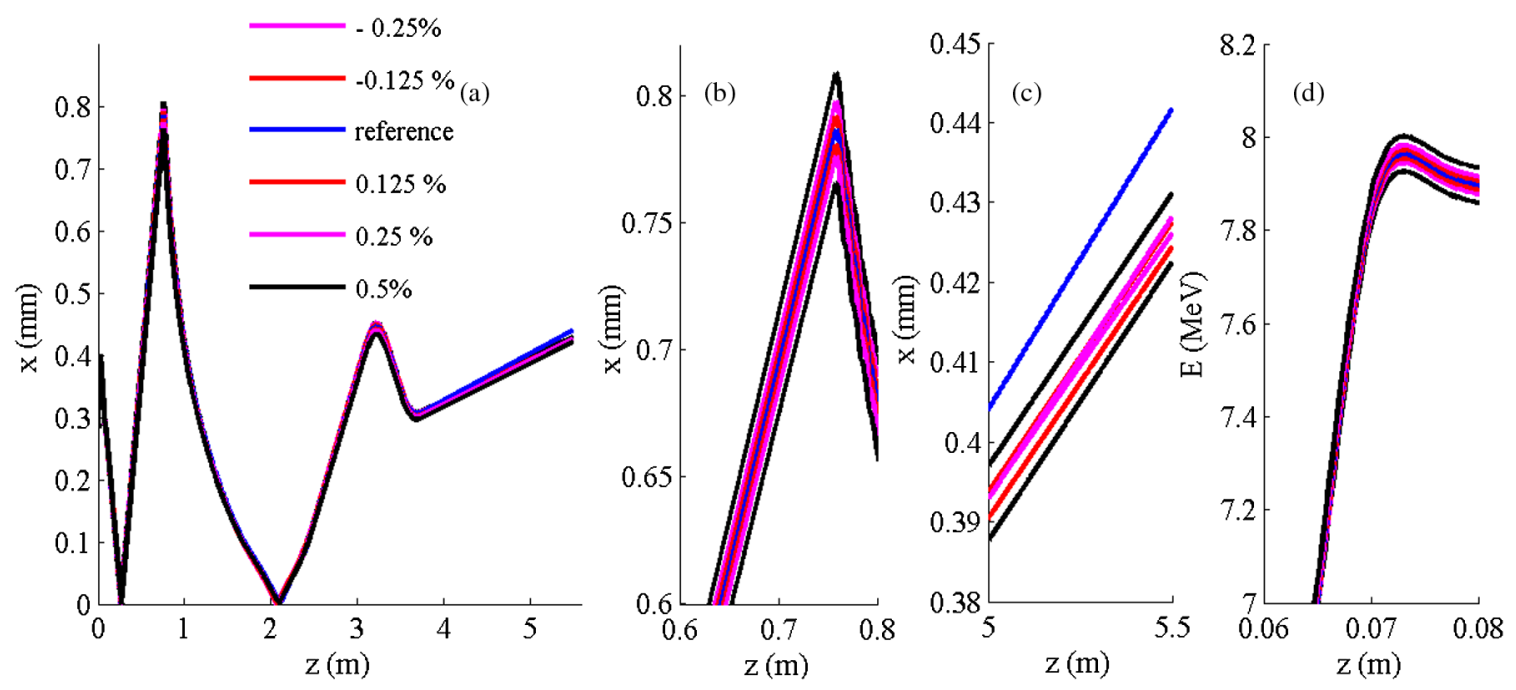

FIG. 33. (a) Simulated trajectories along beamline for various changes in the rf field amplitude-the reference trajectory corresponds to a $200 \mathrm{MV} / \mathrm{m}$ cathode field, a laser offset of $300 \mu \mathrm{m}$ and an $88 \mathrm{MV} / \mathrm{m}$ acceleration gradient. (b-d) Zoomed-in views of the trajectories at two $\mathrm{z}$ locations and the beam energy variation just after the gun.

In absolute terms, rms transverse jitter was $8.2 \mu \mathrm{m}$ and $6.5 \mu \mathrm{m}$ in the horizontal and vertical planes, respectively. Results from simple tracking studies are presented in Fig. 33, which assume the laser is displaced by $300 \mu \mathrm{m}$ with respect to the electrical center of gun cathode (i.e., we typically could not run with the laser centered since this area had low QE). They show that for rf field amplitude variations of a few $10^{-3}$, beam position variations comparable to those measured (as shown in Fig. 33) occur. Note that the effect is very nonlinear and similar transverse position offsets are obtained for $\mathrm{rf}$ amplitude variations of $1.25 \times 10^{-3}$ and $5 \times 10^{-3}$. To get a similar variation in transverse position due to $\mathrm{rf}$ phase jitter, $\mathrm{a}+/-6$ degree change is needed, which is much larger than that observed. The energy at the gun exit scales with the gun gradient, and since the solenoidal focal length scales as the square of the energy, the variation in focal length is twice as large as the field variation.

\section{B. Energy stability}

The bunch energy stability was measured by recording the motion of the beam in the spectrometer. The transverse motion was larger than the transverse beam size (which was not the case upstream of the spectrometer) so this motion

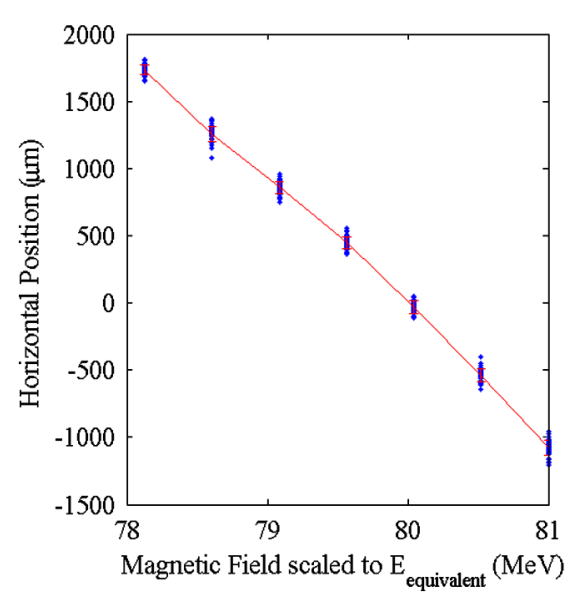

(a)

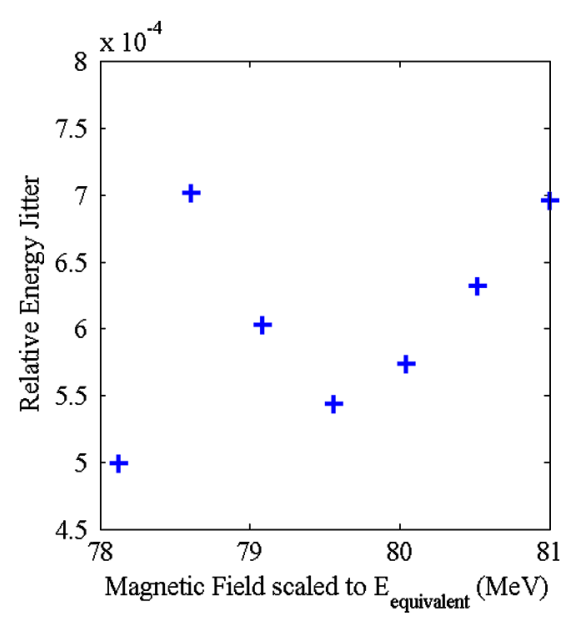

(b)

FIG. 34. Energy stability measured from the beam centroid jitter on the spectrometer screen: (a) 50 samples for each spectrometer magnet setting and (b) energy jitter relative to the nominal $80 \mathrm{MeV}$ beam energy. 
was dominated by energy fluctuations. Note that no vertical motion was observed within the resolution of the screen. The stability of the spectrometer power supply is better than $10^{-4}$ so this was not a factor. The measurements are shown in Fig. 34 where 50 samples were recorded at a $2 \mathrm{~Hz}$ rate for seven settings of spectrometer bend magnet. By varying the magnetic field, we calibrated the position-vs-energy on the screen.

The energy jitter is below $0.1 \% \mathrm{rms}$, which is consistent with the rf field amplitude jitter. The rf phase jitter produces very small energy variations as a 1 degree jitter would generate no more than a $1.4 \times 10^{-4}$ energy jitter when the bunch is on crest.

\section{CONCLUSION}

In summary, we were able to measure the basic performance of an all-X-band injector with the Mark-0 gun, which was originally meant to be used only for debugging purposes. We routinely delivered electron beams up to $85 \mathrm{MeV}$ in a compact system extending over less than $2 \mathrm{~m}$, including a gun and an accelerator powered by a single $\mathrm{X}$-band $(11.424 \mathrm{GHz})$ klystron. The dark current charge was no more than a few tens of $\mathrm{pC}$ downstream of the linac for a cathode field of $200 \mathrm{MV} / \mathrm{m}$. Bunch lengths as short as $125 \mathrm{fs}$ rms were measured for bunch charges of $5 \mathrm{pC}$. Bunch lengths of $465 \mathrm{fs}$ rms were measured for $300 \mathrm{pC}$ bunches, corresponding to a $\sim 300$ A peak current. The thermal emittances measured were comparable to those in S-band guns with $\sim 0.9 \mathrm{~mm}$-mrad per $\mathrm{mm}$ rms laser spot. Emittances measured at $20 \mathrm{pC}$ were typically $0.35 \mathrm{~mm}$-mrad, larger than in the LCLS injector, where they typically are $0.2 \mathrm{~mm}$-mrad. However, in this case our bunch lengths are 3 times shorter than that in the LCLS injector. For a $100 \mathrm{pC}$ bunch charge, the transverse emittance was typically $0.7 \mathrm{~mm}$-mrad, again larger than that in LCLS (typically $0.4 \mathrm{~mm}$-mrad) but our bunch lengths were 3 times shorter. So the peak brightness of our bunches are comparable to those of the LCLS injector for both $20 \mathrm{pC}$ and $100 \mathrm{pC}$ operation.

The laser to electron beam relative stability was less than $50 \mathrm{fs}$ rms if used in a pump-probe configuration [43]. The transverse beam stability was routinely on the order of $10 \%$ of the bunch size. The energy jitter was typically at the $10^{-3}$ level.

As noted, the gun cathode surface was damaged due to laser ablation and $\mathrm{rf}$ breakdown, which made the $\mathrm{QE}$ variation large over the laser spot size. We believe this is the reason why the bunch emittances measured at $30 \mathrm{pC}$ and $100 \mathrm{pC}$ were 2-4 times larger than expected. The lower QE also limited our ability to decrease the laser spot size to try to achieve even lower emittances at low charge.

Overall, we are encouraged by these results and hope that the Mark-1 gun will eventually be tested now that it is much easier to setup and tune the beams at XTA, especially with the aid of several feedback systems that were added. Also, a better support/positioning system for the gun, solenoid and mirror chamber were built but never installed - these would make it much less likely to incur laser damage.

\section{ACKNOWLEDGMENTS}

We thank E. Jongewaard, V. Dolgashev, S. Tantawi, A. Vlieks, and M. Fazio for providing useful information and support, and thank the NLCTA operations staff for their efforts to maintain and improve the facility. We thank the laser group, in particular A. Miahnahri, I. Makasyuk and W. Polzin. Finally, we thank Alan Fischer for helping with instrumentation and calculations for the $\mathrm{THz}$ measurements. This work was supported by the U.S. Department of Energy Contract No. DE-AC02-76SF00515.

[1] M. Altarelli, DESY Report No. 2006-097.

[2] P. Emma, First lasing and operation of an ångstromwavelength free-electron laser, Nat. Photonics 4, 641 (2010).

[3] R. Akre, Commissioning the Linac Coherent Light Source injector, Phys. Rev. ST Accel. Beams 11, 030703 (2008).

[4] I. Hwang, FEL simulation of the 0.1-nm Hard X-ray from the PAL-XFEL, J. Korean Phys. Soc. 64, 212 (2014).

[5] T. Tanaka, Riken Harima Institute SCSS X-FEL Conceptual Design Report, 2005.

[6] R. Gante, Paul Scherrer Institute Swiss FEL CDR Report, 2010.

[7] G. D'Auria, The X-band technology for FELs (XbFEL), 2014.

[8] A. Latina, in International Workshop on Future Linear Colliders (LCWS14), 2014.

[9] Y. Sun, X-band rf driven free electron laser driver with optics linearization, Phys. Rev. ST Accel. Beams 17, 110703 (2014).

[10] C. Adolphsen, Stanford Report No. SLAC-PUB-1124, 2005.

[11] Y. Sun, Low-charge, hard x-ray free electron laser driven with an X-band injector and accelerator, Phys. Rev. ST Accel. Beams 15, 030703, 2012.

[12] W. Leemans, Multi-GeV Electron Beams from CapillaryDischarge-Guided Subpetawatt Laser Pulses in the SelfTrapping Regime, Phys. Rev. Lett. 113, 245002 (2014).

[13] M. Litos, High-efficiency acceleration of an electron beam in a plasma wakefield accelerator, Nature (London) 515, 92 (2014).

[14] A. E. Vlieks, Recent measurements and plans for the SLAC Compton X-ray source, AIP Conf. Proc. 807, 481 (2006).

[15] P. Emma, SLAC National Laboratory Report No. SLACTN, 2001.

[16] V. Dolgashev and J. Wang, RF design of X-band RF deflector for femtosecond diagnostics of LCLS electron beam, AIP Conf. Proc. 1507, 682 (2012).

[17] W. C. Fang, R\&D of X-Band accelerating structure for compact FEL at SINAP, in Proceedings of LINAC 2014 (JACoW, Geneva, Switzerland, 2014).

[18] G. D'Auria, X-Band Technology for FEL sources, in Proceedings of LINAC 2014 (JACoW, Geneva, Switzerland, 2014). 
[19] R. A. Marsh, G. G. Anderson, S. G. Anderson, C. P. J. Barty, and D. J. Gibson, X-Band RF Photoinjector for Laser Compton X-Ray and Gamma-Ray Sources, in Proceedings of 6th International Particle Accelerator Conference (Richmond, Virginia, USA, 2015).

[20] R. Marsh, Modeling and design of an X-band rf photoinjector, Phys. Rev. ST Accel. Beams 15, 102001 (2012).

[21] C. Nantista, Stanford University Report No. SLAC-PUB 6145, 1993.

[22] K. Kim, Rf and space charge effects in laser driven rf electron guns, Nucl. Instrum. Methods Phys. Res., Sect. A 275, 201 (1989).

[23] D. Filipetto, Maximum current density and beam brightness achievable by laser-driven electron sources, Phys. Rev. ST Accel. Beams 17, 024201 (2014).

[24] R. Li, Nanometer emittance ultralow charge beams from rf photoinjectors, Phys. Rev. ST Accel. Beams 15, 090702 (2012).

[25] K. Floettmann, ASTRA, DESY, Hamburg, Germany.

[26] C. Limborg, in Proceedings of the 8th European Particle Accelerator Conference, Paris, 2002 (EPS-IGA and CERN, Geneva, 2002).

[27] T. Vecchione, Simulating single crystal copper photocathode emittance, in Proceedings of FEL 2015 (Daejeon, Korea, 2015).

[28] R. H. Fowler and L. Nordheim, Electron Emission in Intense Electric Fields, in Proceedings of the Royal Society of London. Series A, Containing Papers of a Mathematical and Physical Character, Vol. 119 (The Royal Society, 1928), pp. 173-181.

[29] L. Laurent, Pulsed RF Breakdown studies Report No. SLAC-PUB-8409, 2000.

[30] C. Adolphsen, Stanford University Report No. SLACPUB-9906, 2003.

[31] S. B. van der Geer and M. J. de Loos, General Particle Tracer, GPT, http://www.pulsar.nl/gpt.

[32] J. C. Rosenzweig, in 1995 Particle Accelerator Conference (IEEE Publishing, Piscataway, New Jersey, 1996).

[33] C. K. Allen and N. D. Pattengale, Los Alamos National Laboratory, Report No. LA-UR-02-4979 (unpublished).
[34] C. Limborg et al., A modified quadscan technique for emittance measurement of space charge dominated beams, in Proceedings Particle Accelerator Conference (2006).

[35] V. Dolgashev, Design and application of multimegawatt X-band deflectors for femtosecond electron beam diagnostics, Phys. Rev. ST Accel. Beams 17, 102801 (2014).

[36] T. Van, Oudheusden, Compression of Subrelativistic Space-Charge-Dominated Electron Bunches for SingleShot Femtosecond Electron Diffractionon, Phys. Rev. Lett. 105, 264801 (2010).

[37] C. P. Hauri, Intrinsic Emittance Reduction of an Electron Beam from Metal Photocathodes, Phys. Rev. Lett. 104, 234802 (2010).

[38] H. G. Qiang, Experimental investigation of thermal emittance components of copper photocathode, Phys. Rev. ST Accel. Beams 15, 040102 (2012).

[39] Y. Ding et al., Measurements and simulations of ultra-low emittance and ultra-short electron beams in the linac coherent light source, Phys. Rev. Lett. 102, 254801 (2009).

[40] D. Dowell and J. Schmerge, Quantum efficiency and thermal emittance of metal photocathodes, Phys. Rev. ST Accel. Beams 12, 119901 (2009).

[41] H. Li, C. Limborg-Deprey, C. Adolphsen, D. McCormick, M. Dunning, K. Jobe, T. Raubenheimer, A. Vrielink, T. Vecchione, F. Wang, and S. Weathersby, Two-photon photoemission from a copper cathode in a compact X-band photo injector, Phys. Rev. Accel. Beams 19, 023401 (2016).

[42] C. Limborg, Maximizing brightness in photo injectors, in The Physics and Applications of High Brightness Beams edited by L. Palumbo, J. Rosenzweig and L. Serafini (World Scientific, 2005).

[43] C. Limborg-Deprey et al., An X-Band Photoelectron RF Gun for an Ultrafast Electron Diffraction Source, in Proceedings of the Conference on Femtosecond Electron Imaging and Spectroscopy, FEIS 2013, Key West, FL, USA, edited by P. W. Hawkes, Advances in Imaging and Electron Physics Vol. 191 (Academic Press, UK, 2015. 